\title{
Full Algebra of Generalized Functions and Non-Standard Asymptotic Analysis
}

\author{
Todor D. Todorov* \\ Mathematics Department \\ California Polytechnic State University \\ San Luis Obispo, California 93407, USA \\ (ttodorov@calpoly.edu) \\ Hans Vernaeve* \\ Unit for Engineering Mathematics \\ University of Innsbruck, A-6020 Innsbruck, Austria \\ (hans.vernaeve@uibk.ac.at)
}

\begin{abstract}
We construct an algebra of generalized functions endowed with a canonical embedding of the space of Schwartz distributions. We offer a solution to the problem of multiplication of Schwartz distributions similar to but different from Colombeau's solution. We show that the set of scalars of our algebra is an algebraically closed field unlike its counterpart in Colombeau theory, which is a ring with zero divisors. We prove a Hahn-Banach extension principle which does not hold in Colombeau theory. We establish a connection between our theory with non-standard analysis and thus answer, although indirectly, a question raised by J.F. Colombeau. This article provides a bridge between Colombeau theory of generalized functions and non-standard analysis.
\end{abstract}

*Both authors were partly supported by START-project Y237 of the Austrian Science Fund. The second author was also supported by research grant M949 of Austrian Science Fund.

Mathematics Subject Classification. Primary: 46F30; Secondary: 46F10, 03H05, 46S20, 46S10, 35D05. 
Key words: Schwartz distributions, generalized functions, Colombeau algebra, multiplication of distributions, non-standard analysis, infinitesimals, Robinson valuation field, ultra-metric, Hahn-Banach theorem.

\section{Introduction}

In the early 70's, A. Robinson introduced a real closed, non-archimedean field $\rho^{R}$ (Robinson [32]) as a factor ring of non-standard numbers in ${ }^{*} \mathbb{R}$ (Robinson [31]). The field ${ }^{\rho} \mathbb{R}$ is known as Robinson field of asymptotic numbers (or Robinson valuation field), because it is a natural framework of the classical asymptotic analysis (Lightstone \& Robinson [20]). Later W.A.J. Luxemburg [23] established a connection between ${ }^{\rho} \mathbb{R}$ and $p$-adic analysis (see also the beginning of Section 8 in this article). Li Bang-He [19] studied the connection between ${ }^{\rho} \mathbb{R}$ and the analytic representation of Schwartz distributions, and V. Pestov [29] involved the field ${ }^{\rho} \mathbb{R}$ and similar constructions in the theory of Banach spaces. More recently, it was shown that the field $\rho \mathbb{R}$ is isomorphic to a particular Hahn field of generalized power series (Todorov \& Wolf [37]). The algebras ${ }^{\rho} \mathcal{E}(\Omega)$ of $\rho$-asymptotic functions were introduced in (Oberguggenberger \& Todorov [27]) and studied in Todorov [36]. It is a differential algebra over Robinson's field ${ }^{\rho} \mathbb{C}$ containing a copy of the Schwartz distributions $\mathcal{D}^{\prime}(\Omega)$ (Vladimirov [40]). Applications of $\rho \mathcal{E}(\Omega)$ to partial differential equations were presented in Oberguggenberger [26]. We sometimes refer to the mathematics associated directly or indirectly with the fields ${ }^{\rho} \mathbb{R}$ as non-standard asymptotic analysis.

On the other hand, in the early 80's, J.F. Colombeau developed his theory of new generalized functions without any connection, at least initially, with nonstandard analysis (Colombeau [6]-[10]). This theory is known as Colombeau theory or non-linear theory of generalized functions because it solves the problem of the multiplication of Schwartz distributions. Here is a summary of Colombeau theory presented in axiomatic like fashion: Let $\mathcal{T}^{d}$ denote the usual topology on $\mathbb{R}^{d}$ and let $G$ be an open set of $\mathbb{R}^{d}$. A set $\mathcal{G}(G)$ is called a special algebra of generalized functions on $G$ (of Colombeau type) if there exists a family $\mathcal{G}=:\{\mathcal{G}(\Omega)\}_{\Omega \in \mathcal{T}^{d}}$ (we use =: for "equal by definition") such that:

1. Each $\mathcal{G}(\Omega)$ is a commutative differential ring, i.e. $\mathcal{G}(\Omega)$ is a commutative ring supplied with partial derivatives $\partial^{\alpha}, \alpha \in \mathbb{N}_{0}^{d}$ (linear operators obeying the chain rule). Here $\mathbb{N}_{0}=\{0,1,2, \ldots\}$. Let $\overline{\mathbb{C}}$ denote the ring of generalized scalars of the family $\mathcal{G}$ defined as the set of the functions in $\mathcal{G}\left(\mathbb{R}^{d}\right)$ with zero gradient. Each $\mathcal{G}(\Omega)$ becomes a differential algebra over the ring $\overline{\mathbb{C}}$ (hence, the terminology Colombeau algebras, for short). 
2. The ring of generalized scalars $\overline{\mathbb{C}}$ is of the form $\overline{\mathbb{C}}=\overline{\mathbb{R}} \oplus i \overline{\mathbb{R}}$, where $\overline{\mathbb{R}}$ is a partially ordered real ring, which is a proper extension of $\mathbb{R}$. (Real ring means a ring with the property that $a_{1}^{2}+a_{1}^{2}+\cdots+a_{n}^{2}=0$ implies $a_{1}=a_{2}=\cdots=a_{n}=0$ ). The formula $|x+i y|=\sqrt{x^{2}+y^{2}}$ defines an absolute value on $\overline{\mathbb{C}}$. Consequently, $\overline{\mathbb{C}}$ is a proper extension of $\mathbb{C}$ and both $\overline{\mathbb{R}}$ and $\overline{\mathbb{C}}$ contain non-zero infinitesimals. In Colombeau theory the infinitesimal relation $\approx$ in $\overline{\mathbb{C}}$ is called association.

3. $\overline{\mathbb{C}}$ is spherically complete under some ultra-metric $d_{v}$ on $\overline{\mathbb{C}}$ which agrees with the partial order in $\overline{\mathbb{R}}$ in the sense that $\left|z_{1}\right|<\left|z_{2}\right|$ implies $d_{v}\left(0, z_{1}\right) \leq d_{v}\left(0, z_{2}\right)$.

4. For every $f \in \mathcal{G}(\Omega)$ and every test function $\tau \in \mathcal{D}(\Omega)$ a pairing $(f \mid \tau) \in \overline{\mathbb{C}}$ is defined (with the usual linear properties). Here $\mathcal{D}(\Omega)$ stands for the class of $\mathcal{C}^{\infty}$-functions from $\Omega$ to $\mathbb{C}$ with compact supports. Let $f, g \in \mathcal{G}(\Omega)$. The functions $f$ and $g$ are called weakly equal (or equal in the sense of generalized distributions), in symbol $f \cong g$, if $(f \mid \tau)=(g \mid \tau)$ for all $\tau \in \mathcal{D}(\Omega)$. Similarly, $f$ and $g$ are weakly associated (or simply, associated, for short), in symbol $f \approx g$, if $(f \mid \tau) \approx(g \mid \tau)$ for all $\tau \in \mathcal{D}(\Omega)$, where $\approx$ in the latter formula stands for the infinitesimal relation in $\overline{\mathbb{C}}$.

5. The family $\mathcal{G}$ is a sheaf. That means that $\mathcal{G}$ is supplied with a restriction $\uparrow$ to an open set (with the usual sheaf properties, cf. A. Kaneko [16]) such that $\mathcal{T}^{d} \ni \mathcal{O} \subseteq \Omega$ and $f \in \mathcal{G}(\Omega)$ implies $f \uparrow \mathcal{O} \in \mathcal{G}(\mathcal{O})$. Consequently, each generalized function $f \in \mathcal{G}(\Omega)$ has a support $\operatorname{supp}(f)$ which is a closed subset of $\Omega$.

6. Let $\Omega, \Omega^{\prime} \in \mathcal{T}^{d}$ and $\operatorname{Diff}\left(\Omega^{\prime}, \Omega\right)$ denote the set of all $\mathcal{C}^{\infty}$-diffeomorphisms from $\Omega^{\prime}$ to $\Omega\left(\mathcal{C}^{\infty}\right.$-bijections with $\mathcal{C}^{\infty}$-inverse). A composition (change of variables) $f \circ \psi \in \mathcal{G}\left(\Omega^{\prime}\right)$ is defined for all $f \in \mathcal{G}(\Omega)$ and all $\psi \in \operatorname{Diff}\left(\Omega^{\prime}, \Omega\right)$.

7. For every $\Omega \in \mathcal{T}^{d}$ there exists an embedding $E_{\Omega}: \mathcal{D}^{\prime}(\Omega) \rightarrow \mathcal{G}(\Omega)$ of the space of Schwartz distributions $\mathcal{D}^{\prime}(\Omega)$ into $\mathcal{G}(\Omega)$ such that:

(a) $E_{\Omega}$ preserves the vector operations and partial differentiation in $\mathcal{D}^{\prime}(\Omega)$;

(b) $E_{\Omega}$ is sheaf-preserving, i.e. $E_{\Omega}$ preserves the restriction to open sets. Consequently, $E_{\Omega}$ preserves the support of the Schwartz distributions.

(c) $E_{\Omega}$ preserves the ring operations and partial differentiation in the class $\mathcal{E}(\Omega)$. Here $\mathcal{E}(\Omega)$ stands for the class of $\mathcal{C}^{\infty}$-functions from $\Omega$ to $\mathbb{C}$ (where $\mathcal{E}(\Omega)$ is treated as a subspace of $\mathcal{D}^{\prime}(\Omega)$ ). 
(d) $E_{\Omega}$ preserves the pairing between $\mathcal{D}^{\prime}(\Omega)$ and the class of test functions $\mathcal{D}(\Omega)$. Consequently, $E_{\Omega}$ preserves weakly the Schwartz multiplication in $\mathcal{D}^{\prime}(\Omega)$ (multiplication by duality).

(e) $E_{\Omega}$ preserves the usual multiplication in the class of continuous functions $\mathcal{C}(\Omega)$ up to functions in $\mathcal{G}(\Omega)$ that are weakly associated to zero.

(f) $E_{\Omega}$ preserves weakly the composition with diffeomorphisms (change of variables) in the sense that for every $\Omega, \Omega^{\prime} \in \mathcal{T}^{d}$, every $T \in \mathcal{D}^{\prime}(\Omega)$ and every $\psi \in \operatorname{Diff}\left(\Omega^{\prime}, \Omega\right)$ we have $\left(E_{\Omega}(T) \circ \psi \mid \tau\right)=\left(E_{\Omega^{\prime}}(T \circ \psi) \mid \tau\right)$ for all test functions $\tau \in \mathcal{D}\left(\Omega^{\prime}\right)$. Here $T \circ \psi$ stands for the composition in the sense of the distribution theory (V. Vladimirov [40]).

8. A special algebra is called a full algebra of generalized functions (of Colombeau type) if the embedding $E_{\Omega}$ is canonical in the sense that $E_{\Omega}$ can be uniquely determined by properties expressible only in terms which are already involved in the definition of the family $\mathcal{G}=:\{\mathcal{G}(\Omega)\}_{\Omega \in \mathcal{T}^{d}}$.

9. A family $\mathcal{G}=\{\mathcal{G}(\Omega)\}_{\Omega \in \mathcal{T}^{d}}$ of algebras of generalized functions (special or full) is called diffeomorphism-invariant if $E_{\Omega}$ preserves the composition with diffeomorphisms in the sense that $E_{\Omega}(T) \circ \psi=E_{\Omega^{\prime}}(T \circ \psi)$ for all $\Omega, \Omega^{\prime} \in \mathcal{T}^{d}$, all $T \in \mathcal{D}^{\prime}(\Omega)$ and all $\psi \in \operatorname{Diff}\left(\Omega^{\prime}, \Omega\right)$.

We should mention that embeddings $E_{\Omega}$ (canonical or not) of the type described above are, in a sense, optimal in view of the restriction imposed by the Schwartz impossibility results (Schwartz [34]). For a discussion on the topic we refer to (Colombeau [10], p. 8). Every family of algebras $\mathcal{G}(\Omega)$ (special or full) of the type described above offers a solution to the problem of the multiplication of Schwartz distributions because the Schwartz distributions can be multiplied within an associative and commutative differential algebra.

Full algebras of generalized functions were constructed first by J. F. Colombeau [6]. Several years later, in an attempt to simplify Colombeau's original construction J.F. Colombeau and A.Y. Le Roux [8] (and other authors, H. A. Biagioni [2]) defined the so called simple algebras of generalized functions. Later M. Oberguggenberger ([25], Ch.III, §9) proved that the simple algebras are, actually, special algebras in the sense explained above. Diffeomorphism invariant full algebras were developed in (Grosser, Kunzinger, Oberguggenberger \& Steinbauer [13]-[14]). The sets of generalized scalars of all these algebras are rings with zero divisors (Colombeau [6], pp. 136). The algebras of $\rho$-asymptotic functions ${ }^{\rho} \mathcal{E}(\Omega)$ [27], mentioned earlier, are special algebras of Colombeau type with set of generalized scalars which is an algebraically closed field. The counterpart of the embedding $E_{\Omega}$ in [27] is denoted by $\Sigma_{D, \Omega}$. It is certainly 
not canonical because the existence of $\Sigma_{D, \Omega}$ is proved in [27] by saturation principle (in a non-standard analysis framework) and then "fixed by hand" (see Remark 7.9). Among other things the purpose of this article is to construct a canonical embedding $E_{\Omega}$ in ${ }^{\rho} \mathcal{E}(\Omega)$. We achieve this by means of the choice of a particular ultra-power non-standard model (Section 6) and a particular choice of the positive infinitesimal $\rho$ within this model (Definition 6.1, \#12).

Colombeau theory has numerous applications to ordinary and partial differential equations, the theory of elasticity, fluid mechanics, theory of shock waves (Colombeau[6]-[10], Oberguggenberger [25]), to differential geometry and relativity theory (Grosser, Kunzinger, Oberguggenberger \& Steinbauer [14]) and, more recently, to quantum field theory (Colombeau, Gsponer \& Perrot [11]).

Despite the remarkable achievement and promising applications the theory of Colombeau has some features which can be certainly improved. Here are some of them:

(a) The ring of generalized scalars $\overline{\mathbb{C}}$ and the algebras of generalized functions $\mathcal{G}(\Omega)$ in Colombeau theory are constructed as factor rings within the ultrapowers $\mathbb{C}^{I}$ and $\mathcal{E}(\Omega)^{I}$, respectively, for a particular index set $I$. The rings of nets such as $\mathbb{C}^{I}$ and $\mathcal{E}(\Omega)^{I}$ however (as well their subrings) lack general theoretical principles similar to the axioms of $\mathbb{R}$ and $\mathbb{C}$, for example. Neither $\mathbb{C}^{I}$ and $\mathcal{E}(\Omega)^{I}$ are endowed with principles such as the transfer principle or internal definition principle in non-standard analysis. For that reason Colombeau theory has not been able so far to get rid of the index set $I$ even after the factorization which transforms $\mathbb{C}^{I}$ and $\mathcal{E}(\Omega)^{I}$ into $\overline{\mathbb{C}}$ and $\mathcal{G}(\Omega)$, respectively. As a result Colombeau theory remains overly constructive: there are too many technical parameters (with origin in the index set $I$ ) and too many quantifiers in the definitions and theorems. To a certain extent, Colombeau theory resembles what would be the real analysis if it was based not on the axioms of the reals $\mathbb{R}$ but rather on Cauchy's construction of the real numbers as equivalence classes of fundamental sequences in $\mathbb{Q}$.

(b) In a recent article M. Oberguggenberger and H. Vernaeve [28] defined the concept of internal sets of $\overline{\mathbb{C}}$ and $\mathcal{G}(\Omega)$ and showed that theoretical principles similar to order completeness, underflow and overflow principles and saturation principle for internal sets of $\overline{\mathbb{C}}$ and $\mathcal{G}(\Omega)$ hold in Colombeau theory as well although in more restrictive sense compared with non-standard analysis. However the sets of generalized scalars for $\overline{\mathbb{R}}$ and $\overline{\mathbb{C}}$ are still rings with zero divisors and $\overline{\mathbb{R}}$ is only a partially ordered (not totally ordered) ring. These facts lead to technical complications. For example Hahn-Banach extension principles do 
not hold in Colombeau theory (Vernaeve [39]).

\section{In this article:}

(i) We construct a family of algebras of generalized functions $\widehat{\mathcal{E}(\Omega)^{\mathcal{D}}}$ called asymptotic functions (Section 4 ). We show that $\widehat{\mathcal{E}(\Omega)^{\mathcal{D}}}$ are full algebras of Colombeau type (Section 5) in the sense explained above. Thus we offer a solution to the problem of the multiplication of Schwartz distributions similar to but different from Colombeau's solution (Colombeau [6]). Since the full algebras are commonly considered to be more naturally connected to the theory of Schwartz distributions than the special algebras, we look upon $\widehat{\mathcal{E}(\Omega)^{\mathcal{D}}}$ as an improved alternative to the algebra of $\rho$-asymptotic functions ${ }^{\rho} \mathcal{E}(\Omega)$ defined in [27].

(ii) We believe that our theory is a modified and improved alternative to the original Colombeau theory for the following reasons: (a) The set of scalars $\widehat{\mathbb{C}^{\mathcal{D}_{0}}}$ of the algebra $\widehat{\mathcal{E}(\Omega)^{\mathcal{D}_{0}}}$, called here asymptotic numbers, is an algebraically closed field (Theorem 4.2). Recall for comparison that its counterpart in Colombeau theory $\overline{\mathbb{C}}$ is a ring with zero divisors (Colombeau [6], pp. 136). (b) As a consequence we show that a Hahn-Banach extension principle holds for linear functionals with values in $\widehat{\mathbb{C}^{\mathcal{D}_{0}}}$ (Section 86). This result does not have a counterpart in Colombeau theory (Vernaeve [39]). (c) At this stage the construction of $\widehat{\mathcal{E}(\Omega)^{\mathcal{D}}}$ is already simpler than its counterpart in Colombeau [6]; our theory has one (regularization) parameter less.

(iii) Our next goal is to simplify our theory even more by establishing a connection with non-standard analysis (Section 7). For this purpose we construct a particular ultrapower non-standard model called in this article the distributional non-standard model (Section [6). Then we replace the rings of nets $\mathbb{C}^{I}$ and $\mathcal{E}(\Omega)^{I}$ in Colombeau theory by the non-standard ${ }^{*} \mathbb{C}$ and $* \mathcal{E}(\Omega)$, respectively and the regularization parameter $\varepsilon$ in Colombeau theory by a particular (canonical) infinitesimal $\rho$ in $* \mathbb{R}$. We show that the field of asymptotic numbers $\widehat{\mathbb{C}^{\mathcal{D}_{0}}}$ (defined in Section 4) is isomorphic to a particular Robinson field ${ }^{\rho} \mathbb{C}$ (Robinson [32]). We also prove that the algebra of asymptotic functions $\widehat{\mathcal{E}(\Omega)^{\mathcal{D}_{0}}}$ (defined in Section 4) is isomorphic to a particular algebra of $\rho$-asymptotic functions ${ }^{\rho} \mathcal{E}(\Omega)$ introduced in (Oberguggenberger \& Todorov[27]) in the framework of non-standard analysis.

(iv) Among other things this article provides a bridge between Colombeau theory of generalized functions and non-standard analysis and we hope that it will be 
beneficial for both. After all Robinson's non-standard analysis (Robinson [31]) is historically at least several decades older than Colombeau theory. A lot of work had been already done in the non-standard setting on topics similar to those which appear in Colombeau theory. By establishing a connection with non-standard analysis we answer, although indirectly, a question raised by J.F. Colombeau himself in one of his "research projects" (Colombeau [10], pp. 5).

Since the article establishes a connection between two different fields of mathematics, it is written mostly with two types of readers in mind.The readers with background in non-standard analysis might find in Section 2 5 and Section 8 (along with the axiomatic summary of Colombeau theory presented above) a short introduction to the non-linear theory of generalized functions. Notice however that in these sections we do not present the original Colombeau theory but rather a modified (and improved) version of this theory. The reader without background in non-standard analysis will find in Section [6 a short introduction to the subject. The reading of Sections 2,5 does not require background in non-standard analysis.

\section{Ultrafilter on Test Functions}

In this section we define a particular ultrafilter on the class of test functions $\mathcal{D}\left(\mathbb{R}^{d}\right)$ closely related to Colombeau theory of generalized functions (Colombeau [6]). We shall often use the shorter notation $\mathcal{D}_{0}$ instead of $\mathcal{D}\left(\mathbb{R}^{d}\right)$.

In what follows we denote by $R_{\varphi}$ the radius of support of $\varphi \in \mathcal{D}\left(\mathbb{R}^{d}\right)$ defined by

$$
R_{\varphi}= \begin{cases}\sup \left\{\|x\|: x \in \mathbb{R}^{d}, \varphi(x) \neq 0\right\}, & \varphi \neq 0, \\ 1, & \varphi=0 .\end{cases}
$$

2.1 Definition (Directing Sets). We define the directing sequence of sets $\mathcal{D}_{0}, \mathcal{D}_{1}, \mathcal{D}_{2} \ldots$ by letting $\mathcal{D}_{0}=\mathcal{D}\left(\mathbb{R}^{d}\right)$ and 


$$
\begin{aligned}
\mathcal{D}_{n}=\{ & \varphi \in \mathcal{D}\left(\mathbb{R}^{d}\right): \\
& \varphi \text { is real-valued, } \\
& \left(\forall x \in \mathbb{R}^{d}\right)(\varphi(-x)=\varphi(x)), \\
& R_{\varphi} \leq 1 / n \\
& \int_{\mathbb{R}^{d}} \varphi(x) d x=1, \\
& \left(\forall \alpha \in \mathbb{N}_{0}^{d}\right)\left(1 \leq|\alpha| \leq n \Rightarrow \int_{\mathbb{R}^{d}} x^{\alpha} \varphi(x) d x=0\right), \\
& \int_{\mathbb{R}^{d}}|\varphi(x)| d x \leq 1+\frac{1}{n}, \\
& \left.\left(\forall \alpha \in \mathbb{N}_{0}^{d}\right)\left(|\alpha| \leq n \Rightarrow \sup _{x \in \mathbb{R}^{d}}\left|\partial^{\alpha} \varphi(x)\right| \leq\left(R_{\varphi}\right)^{-2(|\alpha|+d)}\right)\right\}, n=1,2, \ldots
\end{aligned}
$$

2.2 Theorem (Base for a Filter). The directing sequence $\left(\mathcal{D}_{n}\right)$ is a base for a free filter on $\mathcal{D}_{0}$ in the sense that

(i) $\mathcal{D}\left(\mathbb{R}^{d}\right)=\mathcal{D}_{0} \supseteq \mathcal{D}_{1} \supseteq \mathcal{D}_{2} \supseteq \mathcal{D}_{3} \supseteq \ldots$

(ii) $\mathcal{D}_{n} \neq \varnothing$ for all $n \in \mathbb{N}$.

(iii) $\bigcap_{n=0}^{\infty} \mathcal{D}_{n}=\varnothing$.

Proof. (i) Clear.

(ii) Let $\varphi_{0} \in \mathcal{D}(\mathbb{R})$ be the test function

$$
\varphi_{0}(x)= \begin{cases}\frac{1}{c} \exp \left(-\frac{1}{1-x^{2}}\right), & -1 \leq x \leq 1, \\ 0, & \text { otherwise }\end{cases}
$$

where $c=\int_{-1}^{1} \exp \left(-\frac{1}{1-x^{2}}\right) d x$. We let $C_{k}=: \sup _{x \in \mathbb{R}}\left|\frac{d^{k}}{d x^{k}} \varphi_{0}(x)\right|$ for each $k \in \mathbb{N}_{0}$ and 
also $C_{\alpha}=C_{\alpha_{1}} \cdots C_{\alpha_{d}}$ for each multi-index $\alpha \in \mathbb{N}_{0}^{d}$. For each $n, m \in \mathbb{N}$ we let

$$
\begin{aligned}
\mathcal{B}_{n, m, d}=\{ & \varphi \in \mathcal{D}\left(\mathbb{R}^{d}\right): \\
& \varphi \text { is real-valued, } \\
& \varphi(-x)=\varphi(x) \text { for all } x \in \mathbb{R}^{d}, \\
& R_{\varphi}=\sqrt{d}, \\
& \int_{\mathbb{R}^{d}} \varphi(x) d x=1, \\
& \int_{\mathbb{R}^{d}} x^{\alpha} \varphi(x) d x=0 \text { for all } \alpha \in \mathbb{N}^{d} \text { with } 1 \leq|\alpha| \leq n, \\
& \int_{\mathbb{R}^{d}}|\varphi(x)| d x \leq \exp \left(\frac{3 d}{m-1}\right), \\
& \left.\sup _{x \in \mathbb{R}^{d}}\left|\partial^{\alpha} \varphi(x)\right| \leq C_{\alpha}\left(2^{d} m^{|\alpha|+d}\right)^{n} \text { for all } \alpha \in \mathbb{N}_{0}^{d}\right\} .
\end{aligned}
$$

Step 1. We show that, if $m>2$, then $\mathcal{B}_{n, m, d} \neq \varnothing$. Let first $d=1$. Then $\varphi_{0} \in \mathcal{B}_{0, m, 1}$. By induction on $n$, let $\varphi_{n-1} \in \mathcal{B}_{n-1, m, 1}$. Define $\varphi_{n}(x)=a \varphi_{n-1}(x)+$ $b \varphi_{n-1}(m x)$, for some constants $a, b \in \mathbb{R}$ to be determined. Then

$$
\int_{\mathbb{R}} \varphi_{n}(x) d x=a+\frac{b}{m} \text { and } \int_{\mathbb{R}} x^{n} \varphi_{n}(x) d x=\left(a+\frac{b}{m^{n+1}}\right) \int_{\mathbb{R}} x^{n} \varphi_{n-1}(x) d x .
$$

To ensure that $\varphi_{n} \in \mathcal{B}_{n, m, 1}$, we choose $a+\frac{b}{m}=1$ and $a+\frac{b}{m^{n+1}}=0$. Solving for $a, b$, we find that $a=-\frac{1}{m^{n}-1}<0$ and $b=\frac{m^{n+1}}{m^{n}-1}>0$. Since $a \neq 0$, also $R_{\varphi_{n}}=1$. Further, since $\frac{1+x}{1-x} \leq 1+3 x \leq \exp (3 x)$ if $0 \leq x \leq \frac{1}{3}$, we have

$$
\begin{aligned}
& \int_{\mathbb{R}}\left|\varphi_{n}(x)\right| d x \leq\left(|a|+\frac{|b|}{m}\right) \int_{\mathbb{R}}\left|\varphi_{n-1}(x)\right| d x= \\
& \frac{m^{n}+1}{m^{n}-1} \int_{\mathbb{R}}\left|\varphi_{n-1}(x)\right| d x \leq \exp \left(\frac{3}{m^{n}}\right) \int_{\mathbb{R}}\left|\varphi_{n-1}(x)\right| d x,
\end{aligned}
$$

so inductively,

$$
\int_{\mathbb{R}}\left|\varphi_{n}(x)\right| d x \leq \prod_{j=1}^{n} \exp \left(\frac{3}{m^{j}}\right) \int_{\mathbb{R}}\left|\varphi_{0}(x)\right| d x \leq \exp \left(\sum_{j=1}^{\infty} \frac{3}{m^{j}}\right)=\exp \left(\frac{3}{m-1}\right) .
$$


Further, $|a|+|b| m^{k}=\frac{m^{n+k+1}+1}{m^{n}-1} \leq 2 m^{k+1}$ for $k \geq 0, m>2$ and $n \geq 1$. Thus we have

$$
\begin{aligned}
\sup _{x \in \mathbb{R}}\left|\frac{d^{k}}{d x^{k}} \varphi_{n}(x)\right| & \leq\left(|a|+|b| m^{k}\right) \sup _{x \in \mathbb{R}}\left|\frac{d^{k}}{d x^{k}} \varphi_{n-1}(x)\right| \\
& \leq 2 m^{k+1} C_{k}\left(2 m^{k+1}\right)^{n-1}=C_{k}\left(2 m^{k+1}\right)^{n} .
\end{aligned}
$$

Hence $\varphi_{n} \in \mathcal{B}_{n, m, 1}$. Now let $d \in \mathbb{N}$ and $\varphi \in \mathcal{B}_{n, m, 1}$ arbitrary. We have $\psi(x)=$ : $\varphi\left(x_{1}\right) \cdots \varphi\left(x_{d}\right) \in \mathcal{B}_{n, m, d}$

Step 2. Fix $d \in \mathbb{N}$. Let $n \geq 1$, let $M=\max \left\{1, \max _{|\alpha| \leq n} C_{\alpha}\right\}$, let $\psi \in \mathcal{B}_{n, 9 d n, d}$, let $\varepsilon=\frac{1}{d M(18 d n)^{d n}}$ and let $\varphi(x)=\frac{1}{\varepsilon^{d}} \psi(x / \varepsilon)$. We show that $\varphi \in \mathcal{D}_{n}$. If $\|x\| \geq 1 / n \geq \varepsilon \sqrt{d}$, then $\varphi(x)=0$. Further, since $\exp (x) \leq \frac{1}{1-x}$ if $0 \leq x<1$, we have

$$
\int_{\mathbb{R}^{d}}|\varphi(x)| d x=\int_{\mathbb{R}^{d}}|\psi(x)| d x \leq \exp \left(\frac{3 d}{9 d n-1}\right) \leq 1+\frac{3 d}{9 d n-1-3 d} \leq 1+\frac{1}{n} .
$$

Finally, notice that $R_{\varphi}=\varepsilon R_{\psi}=\varepsilon \sqrt{d}$. Thus for $|\alpha| \leq n$ we have

$$
\begin{aligned}
\sup _{x \in \mathbb{R}^{d}}\left|\partial^{\alpha} \varphi(x)\right| & \leq \varepsilon^{-|\alpha|-d} \sup _{x \in \mathbb{R}^{d}}\left|\partial^{\alpha} \psi(x)\right| \leq \varepsilon^{-|\alpha|-d} C_{\alpha}\left(2^{d}(9 d n)^{|\alpha|+d}\right)^{n} \leq \\
& \leq \varepsilon^{-|\alpha|-d} C_{\alpha}(18 d n)^{d n(|\alpha|+d)}=\varepsilon^{-|\alpha|-d} C_{\alpha}(\varepsilon d M)^{-|\alpha|-d} \leq \\
& \leq C_{\alpha} M^{-1}\left(R_{\varphi}\right)^{-2(|\alpha|+d)} .
\end{aligned}
$$

Hence $\varphi \in \mathcal{D}_{n}$ as required.

(iii) Suppose (on the contrary) that there exists $\varphi \in \bigcap_{n=1}^{\infty} \mathcal{D}_{n}$. That means (among other things) that $\int_{\mathbb{R}^{d}} \varphi(x) x^{\alpha} d x=0$ for all $\alpha \neq 0$. Thus we have $\partial^{\alpha} \widehat{\varphi}(0)=0$ for all $\alpha \neq 0$, where $\widehat{\varphi}$ denotes the Fourier transform of $\varphi$. It follows that $\widehat{\varphi}=C$ for some constant $C \in \mathbb{C}$ since $\widehat{\varphi}$ is an entire function on $\mathbb{C}^{d}$ by the Paley-Wiener Theorem (Bremermann [3], Theorem 8.28, pp. 97). Hence by Fourier inversion, $\varphi=(2 \pi)^{d} C \delta \in \mathcal{D}\left(\mathbb{R}^{d}\right)$, where $\delta$ stands for the Dirac delta function. The latter implies $C=0$, thus $\varphi=0$, contradicting the property $\int_{\mathbb{R}^{d}} \varphi(x) d x=1$ in the definition of $\mathcal{D}_{n}$.

In what follows $\mathfrak{c}=: \operatorname{card}(\mathbb{R})$ and $\mathfrak{c}^{+}$stands for the successor of $\mathfrak{c}$.

2.3 Theorem (Existence of Ultrafilter). There exists a $\mathfrak{c}^{+}$-good ultrafilter (maximal filter) $\mathcal{U}$ on $\mathcal{D}_{0}=: \mathcal{D}\left(\mathbb{R}^{d}\right)$ such that $\mathcal{D}_{n} \in \mathcal{U}$ for all $n \in \mathbb{N}_{0}$ (Definition 2.1).

Proof. We observe that $\operatorname{card}\left(\mathcal{D}_{0}\right)=\mathfrak{c}$. The existence of a (free) ultrafilter containing all $\mathcal{D}_{n}$ follows easily by Zorn's lemma since the set $\mathcal{F}=\left\{A \in \mathcal{P}\left(\mathcal{D}_{0}\right): \mathcal{D}_{n} \subseteq\right.$ $A$ for some $\left.n \in \mathbb{N}_{0}\right\}$ is clearly a free filter on $\mathcal{D}_{0}$. Here $\mathcal{P}\left(\mathcal{D}_{0}\right)$ stands for the power 
set of $\mathcal{D}_{0}$. For the existence of a $\mathfrak{c}^{+}$-good ultrafilter containing $\mathcal{F}$ we refer the reader to (Chang \& Keisler [5]) (for a presentation we also mention the Appendix in Lindstrøm [21]).

Let $\mathcal{U}$ be a $\mathfrak{c}^{+}$-good ultrafilter on $\mathcal{D}_{0}=: \mathcal{D}\left(\mathbb{R}^{d}\right)$ containing all $\mathcal{D}_{n}$. We shall keep $\mathcal{U}$ fixed to the end of this article.

For those readers who are unfamiliar with the used terminology we present a list of the most important properties of $\mathcal{U}$. The properties (1)-(3) below express the fact that $\mathcal{U}$ is a filter, the property (1)-(4) express the fact that $\mathcal{U}$ is a free filter, the property (1)-(5) means that $\mathcal{U}$ is a free ultrafilter (maximal filter) and (6) expresses the property of $\mathcal{U}$ to be $c^{+}$-good.

2.4 Lemma (List of Properties of $\mathcal{U}$ ). The ultrafilter $\mathcal{U}$ is a set of subsets of $\mathcal{D}_{0}=$ $\mathcal{D}\left(\mathbb{R}^{d}\right)$ such that $\mathcal{D}_{n} \in \mathcal{U}$ for all $n \in \mathbb{N}_{0}$ and such that:

1. If $A \in \mathcal{U}$ and $B \subseteq \mathcal{D}_{0}$, then $A \subseteq B$ implies $B \in \mathcal{U}$.

2. $\mathcal{U}$ is closed under finite intersections.

3. $\varnothing \notin \mathcal{U}$.

4. $\mathcal{U}$ is a free filter in the sense that $\cap_{A \in \mathcal{U}} A=\varnothing$.

5. Let $A_{k} \in \mathcal{P}\left(\mathcal{D}_{0}\right), k=1,2, \ldots, n$, for some $n \in \mathbb{N}$. Then $\cup_{k=1}^{n} A_{k} \in \mathcal{U}$ implies $A_{k} \in \mathcal{U}$ for at least one $k$. Moreover, if the sets $A_{k}$ are mutually disjoint, then $\cup_{k=1}^{n} A_{k} \in \mathcal{U}$ implies $A_{k} \in \mathcal{U}$ for exactly one $k$. In particular, for every set $A \in \mathcal{P}\left(\mathcal{D}_{0}\right)$ exactly one of $A \in \mathcal{U}$ or $\mathcal{D}_{0} \backslash A \in \mathcal{U}$ is true. Consequently, $\mathcal{U}$ has the finite intersection property (f.i.p.) since $F \notin \mathcal{U}$ and $\mathcal{D}_{0} \backslash F \in \mathcal{U}$ for every finite set $F$ of $\mathcal{D}_{0}$.

6. $\mathcal{U}$ is $\mathfrak{c}^{+}$-good in the sense that for every set $\Gamma \subseteq \mathcal{D}_{0}$, with $\operatorname{card}(\Gamma) \leq \mathfrak{c}$, and every reversal $R: \mathcal{P}_{\omega}(\Gamma) \rightarrow \mathcal{U}$ there exists a strict reversal $S: \mathcal{P}_{\omega}(\Gamma) \rightarrow \mathcal{U}$ such that $S(X) \subseteq R(X)$ for all $X \in \mathcal{P}_{\omega}(\Gamma)$. Here $\mathcal{P}_{\omega}(\Gamma)$ denotes the set of all finite subsets of $\Gamma$.

Recall that a function $R: \mathcal{P}_{\omega}(\Gamma) \rightarrow \mathcal{U}$ is called a reversal if $X \subseteq Y$ implies $R(X) \supseteq R(Y)$ for every $X, Y \in \mathcal{P}_{\omega}(\Gamma)$. A strict reversal is a function $S: \mathcal{P}_{\omega}(\Gamma) \rightarrow$ $\mathcal{U}$ such that $S(X \cup Y)=S(X) \cap S(Y)$ for every $X, Y \in \mathcal{P}_{\omega}(\Gamma)$. It is clear that every strict reversal is a reversal (which justifies the terminology). 
2.5 Definition (Almost Everywhere). Let $P(x)$ be a predicate in one variable defined on $\mathcal{D}_{0}$ (expressing some property of the test functions). We say that $P(\varphi)$ holds almost everywhere in $\mathcal{D}_{0}$ or, simply, $P(\varphi)$ a.e. (where a.e. stands for "almost everywhere"), if $\left\{\varphi \in \mathcal{D}_{0}: P(\varphi)\right\} \in \mathcal{U}$.

2.6 Example (Radius of Support). Let $R_{\varphi}$ be the support of $\varphi$ (cf. (11)) and let $n \in \mathbb{N}$. Then $\left(R_{\varphi} \in \mathbb{R}_{+} \& R_{\varphi}<1 / n\right)$ a.e. because $\mathcal{D}_{n} \subseteq\left\{\varphi \in \mathcal{D}_{0}: R_{\varphi} \in \mathbb{R}_{+} \& R_{\varphi}<\right.$ $1 / n\}$ implies $\left\{\varphi \in \mathcal{D}_{0}: R_{\varphi} \in \mathbb{R}_{+} \& R_{\varphi}<1 / n\right\} \in \mathcal{U}$ by \#1 of Lemma 2.4.

The justification of the terminology "almost everywhere" is based on the observation that the mapping $\mathcal{M}_{\mathcal{U}}: \mathcal{P}\left(\mathcal{D}_{0}\right) \rightarrow\{0,1\}$, defined by $\mathcal{M}_{\mathcal{U}}(A)=1$ if $A \in \mathcal{U}$ and $\mathcal{M}_{\mathcal{U}}(A)=0$ if $A \notin \mathcal{U}$ is finitely additive probability measure on $\mathcal{D}_{0}$.

\section{$3 \quad \mathcal{D}_{0}$-Nets and Schwartz Distributions}

3.1 Definition (Index Set and Nets). Let $\mathcal{D}_{0}, \mathcal{D}_{1}, \mathcal{D}_{2}, \ldots$ be the directing sequence defined in (Definition 2.1), where $\mathcal{D}_{0}=\mathcal{D}\left(\mathbb{R}^{d}\right)$. Let $S$ be a set. The functions of the form $A: \mathcal{D}_{0} \rightarrow S$ are called $\mathcal{D}_{0}$-nets in $S$ or, simply nets in $S$ for short (Kelley [17, p. 65). We denote by $S^{\mathcal{D}_{0}}$ the set of all $\mathcal{D}_{0}$-nets in $S$. The space of test functions $\mathcal{D}_{0}$ is the index set of the nets. If $A \in S^{\mathcal{D}_{0}}$ is a net in $S$, we shall often write $A_{\varphi}$ and $\left(A_{\varphi}\right)$ instead of $A(\varphi)$ and $A$, respectively.

In this section we present several technical lemmas about $\mathcal{D}_{0}$-nets which are closely related to the Schwartz theory of distributions and the directing sequence $\left(\mathcal{D}_{n}\right)$ (Section 21). Our terminology and notation in distribution theory is close to those in Vladimirov [40]. We start with several examples of $\mathcal{D}_{0}$-nets.

3.2 Examples (Nets and Distributions). 1. We denote by $\mathbb{C}^{\mathcal{D}_{0}}$ the set of all nets of the form $A: \mathcal{D}_{0} \rightarrow \mathbb{C}$. We shall often write $\left(A_{\varphi}\right)$ instead of $A$ for the nets in $\mathbb{C}^{\mathcal{D}_{0}}$. It is clear that $\mathbb{C}^{\mathcal{D}_{0}}$ is a ring with zero divisors under the usual pointwise operations. Notice that the nets in $\mathbb{C}^{\mathcal{D}_{0}}$ can be viewed as complex valued functionals (not necessarily linear) on the space of test functions $\mathcal{D}\left(\mathbb{R}^{d}\right)$.

2. Let $\Omega$ be an open subset of $\mathbb{R}^{d}$ and $\mathcal{E}(\Omega)=: \mathcal{C}^{\infty}(\Omega)$. We denote by $\mathcal{E}(\Omega)^{\mathcal{D}_{0}}$ the set of all nets of the form $f: \mathcal{D}_{0} \rightarrow \mathcal{E}(\Omega)$. We shall often write $\left(f_{\varphi}\right)$ or $\left(f_{\varphi}(x)\right)$ instead of $f$ for the nets in $\mathcal{E}(\Omega)^{\mathcal{D}_{0}}$.

3. Let $S$ be a set and $\mathcal{P}(S)$ stand for the power set of $S$. We denote by $\mathcal{P}(S)^{\mathcal{D}_{0}}$ the set of all nets of the form $\mathcal{A}: \mathcal{D}_{0} \rightarrow \mathcal{P}(S)$. We shall often write $\left(\mathcal{A}_{\varphi}\right)$ instead of $\mathcal{A}$ for the nets in $\mathcal{P}(S)^{\mathcal{D}_{0}}$. 
4. Let $\mathcal{T}^{d}$ denote the usual topology on $\mathbb{R}^{d}$. For every open set $\Omega \in \mathcal{T}^{d}$ we let

$$
\begin{aligned}
& \Omega_{\varphi}=\left\{x \in \Omega \mid d(x, \partial \Omega)>R_{\varphi}\right\}, \\
& \widetilde{\Omega}_{\varphi}=\left\{x \in \Omega \mid d(x, \partial \Omega)>2 R_{\varphi} \&\|x\|<1 / R_{\varphi}\right\},
\end{aligned}
$$

where $d(x, \partial \Omega)$ stands for the Euclidean distance between $x$ and the boundary $\partial \Omega$ of $\Omega$ and $R_{\varphi}$ is defined by (11). Let $\chi_{\Omega, \varphi}: \mathbb{R}^{d} \rightarrow \mathbb{R}$ be the characteristic function of the set $\widetilde{\Omega}_{\varphi}$. The cut-off net $\left(C_{\Omega, \varphi}\right) \in \mathcal{E}\left(\mathbb{R}^{d}\right)^{\mathcal{D}_{0}}$ associated with $\Omega$ is defined by the formula $C_{\Omega, \varphi}=: \chi_{\Omega, \varphi} \star \varphi$, where $\star$ stands for the usual convolution, i.e.

$$
C_{\Omega, \varphi}(x)=\int_{\widetilde{\Omega}_{\varphi}} \varphi(x-t) d t
$$

for all $x \in \mathbb{R}^{d}$ and all $\varphi \in \mathcal{D}_{0}$. Notice that $\operatorname{supp}\left(C_{\Omega, \varphi}\right) \subseteq \Omega_{\varphi}$ (Vladimirov [40], Ch.I, $\S 4,6 . T)$.

5. Let $T \in \mathcal{D}^{\prime}(\Omega)$ be a Schwartz distribution on $\Omega$. The $\varphi$-regularization of $T$ is the net $\left(T_{\varphi}\right) \in \mathcal{E}(\Omega)^{\mathcal{D}_{0}}$ defined by the formula $T_{\varphi}=: T \circledast \varphi$, where $T \circledast \varphi$ is a short notation for $\left(C_{\Omega, \varphi} T\right) \star \varphi$ and $\star$ stands (as before) for the usual convolution. In other words, we have

$$
T_{\varphi}(x)=\left(T(t) \mid C_{\Omega, \varphi}(t) \varphi(x-t)\right),
$$

for all $x \in \Omega$ and all $\varphi \in \mathcal{D}_{0}$. Here $(\cdot \mid \cdot)$ stands for the pairing between $\mathcal{D}^{\prime}(\Omega)$ and $\mathcal{D}(\Omega)$ (Vladimirov [40]).

6. We denote by $L_{\Omega}: \mathcal{L}_{\text {loc }}(\Omega) \rightarrow \mathcal{D}^{\prime}(\Omega)$ the Schwartz embedding of $\mathcal{L}_{\text {loc }}(\Omega)$ into $\mathcal{D}^{\prime}(\Omega)$ defined by $L_{\Omega}(f)=T_{f}$. Here $T_{f} \in \mathcal{D}^{\prime}(\Omega)$ stands for the (regular) distribution with kernel $f$, i.e. $\left(T_{f} \mid \tau\right)=\int_{\Omega} f(x) \tau(x) d x$ for all $\tau \in \mathcal{D}^{\prime}(\Omega)$ (Vladimirov [40]). Also, $\mathcal{L}_{\text {loc }}(\Omega)$ denotes the space of the locally integrable (Lebesgue) functions from $\Omega$ to $\mathbb{C}$. Recall that $L_{\Omega}$ preserves the addition and multiplication by complex numbers. The restriction of $L_{\Omega}$ on $\mathcal{E}(\Omega)$ preserves also the partial differentiation (but not the multiplication). We shall write $f \circledast \varphi$ and $f \star \varphi$ instead of $T_{f} \circledast \varphi$ and $T_{f} \star \varphi$, respectively. Thus for every $f \in \mathcal{L}_{\text {loc }}(\Omega)$, every $\varphi \in \mathcal{D}_{0}$ and every $x \in \Omega$ we have

$$
(f \circledast \varphi)(x)=\int_{\| x-t||<R_{\varphi}} f(t) C_{\Omega, \varphi}(t) \varphi(x-t) d t .
$$

In what follows we shall often write $K \Subset \Omega$ to indicate that $K$ is a compact subset of $\Omega$. 
3.3 Lemma (Localization). Let $\Omega$ be (as before) an open set of $\mathbb{R}^{d}$ and $T \in \mathcal{D}^{\prime}(\Omega)$ be a Schwartz distribution. Then for every compact set $K \subset \Omega$ there exists $n \in \mathbb{N}_{0}$ such that for every $x \in K$ and every $\varphi \in \mathcal{D}_{n}$ we have:

(a) $C_{\Omega, \varphi}(x)=1$.

(b) $(T \circledast \varphi)(x)=(T \star \varphi)(x)$.

(c) Consequently, $(\forall K \Subset \Omega)\left(\forall \alpha \in \mathbb{N}_{0}^{d}\right)\left(\exists n \in \mathbb{N}_{0}\right)(\forall x \in K)\left(\forall \varphi \in \mathcal{D}_{n}\right)$ we have $\partial^{\alpha}(T \circledast \varphi)(x)=\left(\partial^{\alpha} T \circledast \varphi\right)(x)=\left(T \circledast \partial^{\alpha} \varphi\right)(x)$.

Proof. (a) Let $d(K, \partial \Omega)$ denote the Euclidean distance between $K$ and $\partial \Omega$. It suffices to choose $n \in \mathbb{N}$ such that $3 / n<d(K, \partial \Omega)$ and $n>\sup _{x \in K}\|x\|+1$. It follows that $3 R_{\varphi}<d(K, \partial \Omega)$ for all $\varphi \in \mathcal{D}_{n}$ because $R_{\varphi} \leq 1 / n$ holds by the definition of $\mathcal{D}_{n}$. Now (a) follows from the property of the convolution (Vladimirov [40], Ch.I, §4,6.T).

(b) If $K \Subset \Omega$, then there exists $m \in \mathbb{N}$ such that $L=:\{t \in \Omega: d(t, K) \leq 1 / m\} \Subset$ $\Omega$. Hence, by part (a), there exists $n \in \mathbb{N}$ (with $n \geq m$ ) such that $C_{\Omega, \varphi}(x) \varphi(x-t)=$ $\varphi(x-t)$ for all $x \in K$, all $t \in \Omega$ and all $\varphi \in \mathcal{D}_{n}$.

(c) follows directly from (b) bearing in mind that we have $\partial^{\alpha}(T \star \varphi)(x)=\left(\partial^{\alpha} T \star\right.$ $\varphi)(x)=\left(T \star \partial^{\alpha} \varphi\right)(x)$.

3.4 Lemma (Schwartz Distributions). Let $\Omega$ be an open set of $\mathbb{R}^{d}$ and $T \in \mathcal{D}^{\prime}(\Omega)$ be a Schwartz distribution. Then for every compact set $K \subset \Omega$ and every multi-index $\alpha \in$ $\mathbb{N}_{0}^{d}$ there exist $m, n \in \mathbb{N}_{0}$ such that for every $\varphi \in \mathcal{D}_{n}$ we have $\sup _{x \in K}\left|\partial^{\alpha}(T \circledast \varphi)(x)\right| \leq$ $\left(R_{\varphi}\right)^{-m}$.

Proof. Let $K$ and $\alpha$ be chosen arbitrarily. By Lemma 3.3, there exists $q \in \mathbb{N}$ such that $\partial^{\alpha}(T \circledast \varphi)(x)=\left(\partial^{\alpha} T \star \varphi\right)(x)$ for all $x \in K$ and all $\varphi \in \mathcal{D}_{q}$. Let $\mathcal{O}$ be an open relatively compact subset of $\Omega$ containing $K$ and let $k \in \mathbb{N}$ be greater than $1 / d(K, \partial \mathcal{O})$. We observe that $\varphi_{x} \in \mathcal{D}(\mathcal{O})$ for all $x \in K$ and all $\varphi \in \mathcal{D}_{k}$, where $\varphi_{x}(t)=: \varphi(x-t)$. On the other hand, there exist $M \in \mathbb{R}_{+}$and $b \in \mathbb{N}_{0}$ such that $\left|\left(\partial^{\alpha} T \mid \tau\right)\right| \leq M \sum_{|\beta| \leq b} \sup _{t \in \mathcal{O}}\left|\partial^{\beta} \tau(t)\right|$ for all $\tau \in \mathcal{D}(\mathcal{O})$ by the continuity of $\partial^{\alpha} T$. Thus $\left|\left(\partial^{\alpha} T \star \varphi\right)(x)\right|=\left|\left(\partial^{\alpha} T \mid \varphi_{x}(t)\right)\right| \leq M \sum_{|\beta| \leq b} \sup _{t \in \mathbb{R}^{d}}\left|\partial^{\beta} \varphi(t)\right|$ for all $x \in K$ and all $\varphi \in \mathcal{D}_{k}$. With this in mind we choose $m=2(b+d)+1$ and $n \geq \max \{q, k, C, b\}$, where $C=M \sum_{|\beta| \leq b} 1$. Now, for every $x \in K$ and every $\varphi \in \mathcal{D}_{n}$ we have

$$
\left|\partial^{\alpha}(T \circledast \varphi)(x)\right| \leq M \sum_{|\beta| \leq b}\left(R_{\varphi}\right)^{-2(|\beta|+d)} \leq C\left(R_{\varphi}\right)^{-2(b+d)} \leq\left(R_{\varphi}\right)^{-m},
$$

as required, where the last inequality holds because $R_{\varphi} \leq 1 / n$ by the definition of $\mathcal{D}_{n}$ (Definition 2.1) and $1 / n \leq 1 / C$ by the choice of $n$. 
3.5 Lemma ( $\mathcal{C}^{\infty}$-Functions). Let $\Omega$ be an open set of $\mathbb{R}^{d}$ and $f \in \mathcal{E}(\Omega)$ be a $\mathcal{C}^{\infty}$ function. Then for every compact set $K \subset \Omega$, every multi-index $\alpha \in \mathbb{N}_{0}^{d}$ and every $p \in \mathbb{N}$ there exists $n \in \mathbb{N}_{0}$ such that for every $\varphi \in \mathcal{D}_{n}$ we have

$$
\sup _{x \in K}\left|\partial^{\alpha}(f \circledast \varphi)(x)-\partial^{\alpha} f(x)\right| \leq\left(R_{\varphi}\right)^{p} .
$$

Proof. Suppose that $p \in \mathbb{N}, K \Subset \Omega$ and $\alpha \in \mathbb{N}_{0}^{d}$. By Lemma 3.3, there exists $q \in \mathbb{N}_{0}$ such that $\partial^{\alpha}(f \circledast \varphi)(x)=\left(\partial^{\alpha} f \star \varphi\right)(x)$ for all $x \in K$ and all $\varphi \in \mathcal{D}_{q}$. As before, let $\mathcal{O}$ be an open relatively compact subset of $\Omega$ containing $K$ and let $k \in \mathbb{N}$ be greater than $1 / d(K, \partial \mathcal{O})$. Let $n \geq \max \left\{p, q, k, \frac{2 C}{(p+1) !}\right\}$, where $C=: \sum_{|\beta|=p+1} \sup _{\xi \in \mathcal{O}}\left|\left(\partial^{\alpha+\beta} f\right)(\xi)\right|$.

Let $x \in K$ and $\varphi \in \mathcal{D}_{n}$. By involving the definition of the sets $\mathcal{D}_{n}$, we calculate:

$$
\begin{aligned}
& \left|\partial^{\alpha}(f \circledast \varphi)(x)-\partial^{\alpha} f(x)\right|=(\text { Lemma 3.3 and } n \geq q)=\left|\left(\partial^{\alpha} f \star \varphi\right)(x)-\partial^{\alpha} f(x)\right|= \\
& (\text { since } n \geq 1)=\left|\int_{|| y|| \leq R_{\varphi}}\left[\partial^{\alpha} f(x-y)-\partial^{\alpha} f(x)\right] \varphi(y) d y\right|=\left(\begin{array}{c}
\text { Taylor expansion } \\
\text { for some } t \in[0,1]
\end{array}\right)= \\
& |\sum_{|\beta|=1}^{p} \frac{(-1)^{|\beta|} \partial^{\alpha+\beta} f(x)}{|\beta| !} \underbrace{\int_{\| y|| \leq R_{\varphi}} y^{\beta} \varphi(y) d y}_{=0 \text { since } n \geq p}+\frac{(-1)^{p+1}}{(p+1) !} \sum_{|\beta|=p+1} \int_{|| y|| \leq R_{\varphi}} y^{\beta} \varphi(y) \partial^{\alpha+\beta} f(x-y t) d y|= \\
& \quad=\frac{R_{\varphi}^{p+1}}{(p+1) !}\left(C \int_{\|y\| \leq R_{\varphi}}|\varphi(y)| d y\right) \leq \frac{R_{\varphi}^{p+1}}{(p+1) !} C(1+1 / n)<\frac{R_{\varphi}^{p+1}}{(p+1) !} 2 C \leq R_{\varphi}^{p},
\end{aligned}
$$

as required, where the last inequality follows from $R_{\varphi} \leq 1 / n \leq(p+1) ! / 2 C$.

3.6 Lemma (Pairing). Let $\Omega$ be an open set of $\mathbb{R}^{d}, T \in \mathcal{D}^{\prime}(\Omega)$ be a Schwartz distribution and $\tau \in \mathcal{D}(\Omega)$ be a test function. Then for every $p \in \mathbb{N}$ there exists $n \in \mathbb{N}_{0}$ such that for every $\varphi \in \mathcal{D}_{n}$ we have

$$
|(T \circledast \varphi \mid \tau)-(T \mid \tau)| \leq\left(R_{\varphi}\right)^{p} .
$$

Proof. Let $p \in \mathbb{N}$ and let $\mathcal{O}$ be an open relatively compact subset of $\Omega$ containing $\operatorname{supp}(\tau)$. There exist $M \in \mathbb{R}_{+}$and $a \in \mathbb{N}_{0}$ such that $|(T \mid \psi)| \leq M \sum_{|\alpha| \leq a} \sup _{x \in \mathcal{O}}\left|\partial^{\alpha} \psi(x)\right|$ for all $\psi \in \mathcal{D}(\mathcal{O})$ by the continuity of $T$. Also, there exists $q \in \mathbb{N}_{0}$ such that $\left|\partial^{\alpha}(\tau \circledast \varphi)(x)-\partial^{\alpha} \tau(x)\right| \leq\left(R_{\varphi}\right)^{p+1}$ for all $x \in \overline{\mathcal{O}}$, all $|\alpha| \leq a$ and all $\varphi \in \mathcal{D}_{q}$ by Lemma 3.5. We observe as well that there exists $m \in \mathbb{N}_{0}$ such that $\tau \circledast \varphi-\tau \in \mathcal{D}(\mathcal{O})$ whenever $\varphi \in \mathcal{D}_{m}$. Let $\varphi \in \mathcal{D}_{n}$, where $n \geq \max \left\{1, q, m, M \sum_{|\alpha| \leq a} 1\right\}$. Since $\varphi(-x)=\varphi(x)$ for all $x \in \mathbb{R}^{d}$, we have $|(T \circledast \varphi \mid \tau)-(T \mid \tau)|=|(T \mid \tau \circledast \varphi-\tau)| \leq$ $M \sum_{|\alpha| \leq a}\left(R_{\varphi}\right)^{p+1}=\left(R_{\varphi}\right)^{p}\left(R_{\varphi}\right) M\left(\sum_{|\alpha| \leq a} 1\right) \leq\left(R_{\varphi}\right)^{p}$ as required. 


\section{Asymptotic Numbers and Asymptotic Functions}

We define a field $\widehat{\mathbb{C}^{\mathcal{D}_{0}}}$ of asymptotic numbers and the differential algebra of asymptotic functions $\widehat{\mathcal{E}(\Omega)^{\mathcal{D}_{0}}}$ over the field $\widehat{\mathbb{C}^{\mathcal{D}_{0}}}$. No background in non-standard analysis is required of the reader: our framework is still the usual standard analysis. Both $\widehat{\mathbb{C}^{\mathcal{D}_{0}}}$ and $\widehat{\mathcal{E}(\Omega)^{\mathcal{D}}}$, however, do have alternative non-standard representations, but we shall postpone the discussion of the connection with non-standard analysis to Section 7.

The readers who are unfamiliar with the non-linear theory of generalized functions (Colombeau [6]-[10]) might treat this and the next sections as an introduction to a (modified and improved version) of Colombeau theory. The readers who are familiar with Colombeau theory will observe the strong similarity between the construction of $\widehat{\mathbb{C}^{D_{0}}}$ and the definition of the ring $\overline{\mathbb{C}}$ of Colombeau generalized numbers (Colombeau [6], pp. 136). The definition of $\widehat{\mathcal{E}(\Omega)^{\mathcal{D}_{0}}}$ also resembles the definition of the special algebra $\mathcal{G}(\Omega)$ of Colombeau generalized functions (Colombeau [7]). We believe, however, that our asymptotic numbers and asymptotic functions offer an important improvement of Colombeau theory because $\overline{\mathbb{C}^{\mathcal{D}_{0}}}$ is an algebraically closed field (Theorem 4.2 ) in contrast to $\overline{\mathbb{C}}$, which is a ring with zero divisors.

4.1 Definition (Asymptotic Numbers). Let $R_{\varphi}$ be the radius of support of $\varphi$ (cf.(1)).

1. We define the sets of the moderate and negligible nets in $\mathbb{C}^{\mathcal{D}_{0}}$ by

$$
\begin{aligned}
& \mathcal{M}\left(\mathbb{C}^{\mathcal{D}_{0}}\right)=\left\{\left(A_{\varphi}\right) \in \mathbb{C}^{\mathcal{D}_{0}}:(\exists m \in \mathbb{N})\left(\left|A_{\varphi}\right| \leq\left(R_{\varphi}\right)^{-m} \text { a.e. }\right)\right\}, \\
& \mathcal{N}\left(\mathbb{C}^{\mathcal{D}_{0}}\right)=\left\{\left(A_{\varphi}\right) \in \mathbb{C}^{\mathcal{D}_{0}}:(\forall p \in \mathbb{N})\left(\left|A_{\varphi}\right|<\left(R_{\varphi}\right)^{p} \text { a.e. }\right)\right\},
\end{aligned}
$$

respectively, where "a.e" stands for "almost everywhere" (Definition 2.5). We define the factor ring $\widehat{\mathbb{C}^{\mathcal{D}_{0}}}=: \mathcal{M}\left(\mathbb{C}^{\mathcal{D}_{0}}\right) / \mathcal{N}\left(\mathbb{C}^{\mathcal{D}_{0}}\right)$ and we denote by $\widehat{A_{\varphi}} \in \widehat{\mathbb{C}^{\mathcal{D}_{0}}}$ the equivalence class of the net $\left(A_{\varphi}\right) \in \mathcal{M}\left(\mathbb{C}^{\mathcal{D}_{0}}\right)$.

2. If $\mathcal{S} \subseteq \mathbb{C}^{\mathcal{D}_{0}}$, we let $\widehat{\mathcal{S}}=:\left\{\widehat{A_{\varphi}}:\left(A_{\varphi}\right) \in \mathcal{S} \cap \mathcal{M}\left(\mathbb{C}^{\mathcal{D}_{0}}\right)\right\}$. We call the elements of $\widehat{\mathbb{C}^{\mathcal{D}_{0}}}$ complex asymptotic numbers and the elements of $\widehat{\mathbb{R}^{\mathcal{D}_{0}}}$ real asymptotic numbers. We define an order relation on $\widehat{\mathbb{R}^{\mathcal{D}_{0}}}$ as follows: Let $\widehat{A_{\varphi}} \in \widehat{\mathbb{R}^{\mathcal{D}_{0}}}$ and $\widehat{A_{\varphi}} \neq 0$. Then $\widehat{A_{\varphi}}>0$ if $A_{\varphi}>0$ a.e., that is $\left\{\varphi \in \mathcal{D}_{0}: A_{\varphi}>0\right\} \in \mathcal{U}$.

3. We define the embeddings $\mathbb{C} \subset \widehat{\mathbb{C}^{\mathcal{D}_{0}}}$ and $\mathbb{R} \subset \widehat{\mathbb{R}^{\mathcal{D}_{0}}}$ by the constant nets, i.e. by $A \rightarrow \widehat{A}$.

4.2 Theorem (Algebraic Properties). $\widehat{\mathbb{C}^{\mathcal{D}_{0}}}$ is an algebraically closed field, $\widehat{\mathbb{R}^{\mathcal{D}_{0}}}$ is a real closed field and we have the usual connection $\widehat{\mathbb{C}^{\mathcal{D}_{0}}}=\widehat{\mathbb{R}^{\mathcal{D}_{0}}} \oplus i \widehat{\mathbb{R}^{\mathcal{D}_{0}}}$. 
Proof. It is clear that $\widehat{\mathbb{C}^{\mathcal{D}_{0}}}$ is a ring and $\widehat{\mathbb{C}^{\mathcal{D}_{0}}}=\widehat{\mathbb{R}^{\mathcal{D}_{0}}}+i \widehat{\mathbb{R}^{\mathcal{D}_{0}}}$. To show that $\widehat{\mathbb{C}^{\mathcal{D}_{0}}}$ is a field, suppose that $\left(A_{\varphi}\right) \in \mathcal{M}\left(\mathbb{C}^{\mathcal{D}_{0}}\right) \backslash \mathcal{N}\left(\mathbb{C}^{\mathcal{D}_{0}}\right)$. Thus there exist $m, p \in \mathbb{N}$ such that $\Phi=:\left\{\varphi \in \mathcal{D}_{0}:\left(R_{\varphi}\right)^{p} \leq\left|A_{\varphi}\right| \leq\left(R_{\varphi}\right)^{-m}\right\} \in \mathcal{U}$. We define the net $\left(B_{\varphi}\right) \in \mathbb{C}^{\mathcal{D}_{0}}$ by $B_{\varphi}=1 / A_{\varphi}$ if $\varphi \in \Phi$ and $B_{\varphi}=1$ if $\varphi \in \mathcal{D}_{0} \backslash \Phi$. It is clear that $A_{\varphi} B_{\varphi}=1$ a.e. thus $\widehat{A_{\varphi}} \widehat{B_{\varphi}}=1$ as required. To show that $\widehat{\mathbb{C D}^{\mathcal{D}}}$ is an algebraically closed field, let $P(x)=x^{p}+a_{n-1} x^{p-1}+\cdots+a_{0}$ be a polynomial with coefficients in $\widehat{\mathbb{C}^{D_{0}}}$ and degree $p \geq 1$. Since $\widehat{\mathbb{C}^{\mathcal{D}_{0}}}$ is a field, we have assumed without loss of generality that the leading coefficient is 1 . We have $a_{k}=\widehat{A_{\varphi, k}}$, for some moderate nets $\left(A_{\varphi, k}\right)$. Denote $P_{\varphi}(x)=: x^{p}+A_{\varphi, p-1} x^{p-1}+\cdots+A_{\varphi, 0}$ and observe that for every $\varphi \in \mathcal{D}_{0}$ there exists a complex number $X_{\varphi} \in \mathbb{C}$ such that $P_{\varphi}\left(X_{\varphi}\right)=0$ since $\mathbb{C}$ is an algebraically closed field. Thus there exists a net $\left(X_{\varphi}\right) \in \mathbb{C}^{\mathcal{D}_{0}}$ such that $P\left(X_{\varphi}\right)=0$ for all $\varphi \in \mathcal{D}_{0}$. Also the estimation $\left|X_{\varphi}\right| \leq 1+\left|A_{\varphi, p-1}\right|+\cdots+\left|A_{\varphi, 0}\right|$ implies that the net $\left(X_{\varphi}\right)$ is a moderate net. The asymptotic number $\widehat{X_{\varphi}} \in \widehat{\mathbb{C}^{\mathcal{D}_{0}}}$ is the zero of the polynomial $P$ we are looking for because $P\left(\widehat{X}_{\varphi}\right)=\widehat{X}_{\varphi}^{p}+a_{p-1} \widehat{X}_{\varphi}^{p-1}+\cdots+a_{0}=$ ${\widehat{X_{\varphi}}}^{p}+{\widehat{A_{\varphi, p-1}}}_{X_{\varphi}}^{p-1}+\cdots+\widehat{A_{\varphi, 0}}=\widehat{P_{\varphi}\left(X_{\varphi}\right)}=\widehat{0}=0$ as required. The fact that $\widehat{\mathbb{R}^{\mathcal{D}_{0}}}$ is a real closed field follows directly from the fact that $\widehat{\mathbb{C}^{\mathcal{D}_{0}}}$ is an algebraically closed field and the connection $\widehat{\mathbb{C}^{\mathcal{D}_{0}}}=\widehat{\mathbb{R}^{\mathcal{D}_{0}}}+i \widehat{\mathbb{R}^{\mathcal{D}_{0}}}$ (Van Der Waerden [38], Chapter 11).

4.3 Corollary (Total Order). $\widehat{\mathbb{R}^{\mathcal{D}_{0}}}$ is a totally ordered field and we have the following characterization of the order relation: if $a \in \widehat{\mathbb{R}^{\mathcal{D}_{0}}}$ then $a \geq 0$ iff $a=b^{2}$ for some $b \in \widehat{\mathbb{R}^{\mathcal{D}_{0}}}$. Consequently, the mapping $|\cdot|: \widehat{\mathbb{C}^{\mathcal{D}_{0}}} \rightarrow \widehat{\mathbb{R}^{\mathcal{D}_{0}}}$, defined by the formula $|a+i b|=\sqrt{a^{2}+b^{2}}$, is an absolute value on $\widehat{\mathbb{C}^{\mathcal{D}_{0}}}$ (Ribenboim [30], pp.3-6).

Proof. The algebraic operations in any real closed field uniquely determine a total order (Van Der Waerden [38, Chapter 11). Thus the characterization of the order relation in $\widehat{\mathbb{R}^{\mathcal{D}_{0}}}$ follows directly from the fact that $\widehat{\mathbb{R}^{\mathcal{D}_{0}}}$ is a real closed field. The existence of the root $\sqrt{x}$ for any non-negative $x$ in $\widehat{\mathbb{R}^{\mathcal{D}_{0}}}$ also follows from the fact that $\widehat{\mathbb{R}^{\mathcal{D}_{0}}}$ is a real closed field.

4.4 Definition (Infinitesimals, Finite and Infinitely Large). An asymptotic number $z \in \widehat{\mathbb{C}^{\mathcal{D}_{0}}}$ is called infinitesimal, in symbol $z \approx 0$, if $|z|<1 / n$ for all $n \in \mathbb{N}$. Similarly, $z$ is called finite if $|z|<n$ for some $n \in \mathbb{N}$. And $z$ is infinitely large if $n<|z|$ for all $n \in \mathbb{N}$. We denote by $\mathcal{I}\left(\widehat{\mathbb{C}^{\mathcal{D}_{0}}}\right), \mathcal{F}\left(\widehat{\mathbb{C}^{\mathcal{D}_{0}}}\right)$ and $\mathcal{L}\left(\widehat{\mathbb{C}^{\mathcal{D}_{0}}}\right)$ the sets of the infinitesimal, finite and infinitely large numbers in $\widehat{\mathbb{C}^{\mathcal{D}_{0}}}$, respectively. We define the standard part mapping $\widehat{\text { st }}: \mathcal{F}\left(\widehat{\mathbb{C}^{\mathcal{D}_{0}}}\right) \rightarrow \mathbb{C}$ by the formula $\widehat{\operatorname{st}}(z) \approx z$.

The next result shows that both $\widehat{\mathbb{R}^{\mathcal{D}_{0}}}$ and $\widehat{\mathbb{C}^{\mathcal{D}_{0}}}$ are non-archimedean fields in the sense that they contain non-zero infinitesimals. 
4.5 Lemma (Canonical Infinitesimal in $\widehat{\mathbb{R}^{D_{0}}}$ ). Let $R_{\varphi}$ be the radius of support of $\varphi\left(c f\right.$.(1)). Then the asymptotic number $\widehat{\rho}=: \widehat{R_{\varphi}}$ is a positive infinitesimal in $\widehat{\mathbb{R}^{\mathcal{D}_{0}}}$. We call $\hat{\rho}$ the canonical infinitesimal in $\widehat{\mathbb{R}^{\mathcal{D}_{0}}}$ (the choice of the notation $\widehat{\rho}$ will be justified in Section (7).

Proof. We have $0 \leq \hat{\rho}<1 / n$ for all $n \in \mathbb{N}$ because $R_{\varphi} \in \mathbb{R}_{+} \& R_{\varphi}<1 / n$ a.e. (cf. Example 2.6). Also, $\widehat{\rho} \neq 0$ because $\left(R_{\varphi}\right) \notin \mathcal{N}\left(\mathbb{C}^{\mathcal{D}_{0}}\right)$.

4.6 Definition (Topology, Valuation, Ultra-Norm, Ultra-Metric). We supply $\widehat{\mathbb{C}^{\mathcal{D}_{0}}}$ with the order topology, i.e. the product topology inherited from the order topology on $\widehat{\mathbb{R}^{\mathcal{D}_{0}}}$. We define a valuation $v: \widehat{\mathbb{C}^{\mathcal{D}_{0}}} \rightarrow \mathbb{R} \cup\{\infty\}$ on $\widehat{\mathbb{C}^{\mathcal{D}_{0}}}$ by $v(z)=\sup \{q \in$ $\left.\mathbb{Q} \mid z / \widehat{\rho}^{q} \approx 0\right\}$ if $z \neq 0$ and $v(0)=\infty$. We define the ultra-norm $|\cdot|_{v}: \widehat{\mathbb{C}^{\mathcal{D}_{0}}} \rightarrow \mathbb{R}$ by the formula $|z|_{v}=e^{-v(z)}$ (under the convention that $e^{-\infty}=0$ ). The formula $d(a, b)=|a-b|_{v}$ defines an ultra-metric on $\widehat{\mathbb{C}^{\mathcal{D}_{0}}}$.

4.7 Theorem (Ultra-Properties). Let $a, b, c \in \widehat{\mathbb{C}^{\mathcal{D}_{0}}}$. Then

(i) (a) $v(a)=\infty$ iff $a=0$; (b) $v(a b)=v(a)+v(b)$. (c) $v(a+b) \geq \min \{v(a), v(b)\}$; (d) $|a|<|b|$ implies $v(a) \geq v(b)$.

(ii) (a) $|0|_{v}=0,| \pm 1|_{v}=1$, and $|a|_{v}>0$ whenever $a \neq 0$; (b) $|a b|_{v}=|a|_{v}|b|_{v}$; (c) $|a+b|_{v} \leq \max \left\{|a|_{v},|b|_{v}\right\}$ (ultra-norm inequality); (d) $|a|<|b|$ implies $|a|_{v} \leq$ $|b|_{v}$.

(iii) $d(a, b) \leq \max \{d(a, c), d(c, b)\}$ (ultra-metric inequality). Consequently, $\left(\widehat{\mathbb{C}^{\mathcal{D}_{0}}}, d\right)$ and $\left(\widehat{\mathbb{R}^{\mathcal{D}_{0}}}, d\right)$ are ultra-metric spaces.

Proof. The properties (i)-(iii) follow easily from the definition of $v$ and we leave the verification to the reader.

4.8 Remark (Colombeau Theory). The counterpart $\bar{v}$ of $v$ in Colombeau theory is only a pseudo-valuation, not a valuation, in the sense that $\bar{v}$ satisfies the property $\bar{v}(a b) \geq \bar{v}(a)+\bar{v}(b)$, not $v(a b)=v(a)+v(b)$. Consequently, the the counterpart $|\cdot|_{\bar{v}}$ of $|\cdot|_{v}$ in Colombeau theory is pseudo-ultra-metric, not a ultra-metric, in the sense that it satisfies the property $|a b|_{\bar{v}} \leq|a|_{\bar{v}}|b|_{\bar{v}}$, not $|a b|_{v}=|a|_{v}|b|_{v}$. For the concept of classical valuation we refer the reader to (Ribenboim [30]).

4.9 Definition (Asymptotic Functions). Let $\Omega$ be an open set of $\mathbb{R}^{d}$ and $R_{\varphi}$ be the radius of support of $\varphi$ (cf.(1)). 
1. We define the sets of the moderate nets $\mathcal{M}\left(\mathcal{E}(\Omega)^{\mathcal{D}_{0}}\right)$ and negligible nets $\mathcal{N}\left(\mathcal{E}(\Omega)^{\mathcal{D}_{0}}\right)$ of $\mathcal{E}(\Omega)^{\mathcal{D}_{0}}$ by: $\left(f_{\varphi}\right) \in \mathcal{M}\left(\mathcal{E}(\Omega)^{\mathcal{D}_{0}}\right)$ if (by definition)

$$
(\forall K \Subset \Omega)\left(\forall \alpha \in \mathbb{N}^{d}\right)\left(\exists m \in \mathbb{N}_{0}\right)\left(\sup _{x \in K}\left|\partial^{\alpha} f_{\varphi}(x)\right| \leq\left(R_{\varphi}\right)^{-m} \text { a.e. }\right),
$$

and, similarly, $\left(f_{\varphi}\right) \in \mathcal{N}\left(\mathcal{E}(\Omega)^{\mathcal{D}_{0}}\right)$ if (by definition)

$$
(\forall K \Subset \Omega)\left(\forall \alpha \in \mathbb{N}^{d}\right)(\forall p \in \mathbb{N})\left(\sup _{x \in K}\left|\partial^{\alpha} f_{\varphi}(x)\right| \leq\left(R_{\varphi}\right)^{p} \text { a.e. }\right),
$$

respectively. Here $\partial^{\alpha} f_{\varphi}(x)$ stands for the $\alpha$-partial derivative of $f_{\varphi}(x)$ with respect to $x$ and "a.e" stands (as before) for "almost everywhere" (Definition 2.5). We define the factor ring $\widehat{\mathcal{E}(\Omega)^{\mathcal{D}_{0}}}=: \mathcal{M}\left(\mathcal{E}(\Omega)^{\mathcal{D}_{0}}\right) / \mathcal{N}\left(\mathcal{E}(\Omega)^{\mathcal{D}_{0}}\right)$ and we denote by $\widehat{f}_{\varphi} \in \widehat{\mathcal{E}(\Omega)^{\mathcal{D}_{0}}}$ the equivalence class of the net $\left(f_{\varphi}\right) \in \mathcal{M}\left(\mathcal{E}(\Omega)^{\mathcal{D}_{0}}\right)$. We call the elements of $\widehat{\mathcal{E}(\Omega)^{D_{0}}}$ asymptotic functions on $\Omega$. More generally, if $\mathcal{S} \subseteq \mathcal{E}(\Omega)^{\mathcal{D}_{0}}$, we let $\widehat{\mathcal{S}}=:\left\{\widehat{f}_{\varphi}:\left(f_{\varphi}\right) \in \mathcal{S} \cap \mathcal{M}\left(\mathcal{E}(\Omega)^{\mathcal{D}_{0}}\right)\right\}$.

2. We supply $\widehat{\mathcal{E}(\Omega)^{\mathcal{D}}}$ with the ring operations and partial differentiation of any order inherited from $\mathcal{E}(\Omega)$. Also, for every asymptotic number $\widehat{A_{\varphi}} \in \widehat{\mathbb{C}^{\mathcal{D}_{0}}}$ and every asymptotic function $\widehat{f}_{\varphi} \in \widehat{\mathcal{E}(\Omega)^{\mathcal{D}_{0}}}$ we define the product $\widehat{A_{\varphi}} \widehat{f}_{\varphi} \in$ $\widehat{\mathcal{E}(\Omega)^{\mathcal{D}_{0}}}$ by $\widehat{A_{\varphi}} \widehat{f}_{\varphi}=\widehat{A_{\varphi} f_{\varphi}}$.

3. We define the pairing between $\widehat{\mathcal{E}(\Omega)^{\mathcal{D}_{0}}}$ and $\mathcal{D}(\Omega)$ by the formula $\left(\widehat{f}_{\varphi} \mid \tau\right)=$ $\widehat{\left(f_{\varphi} \mid \tau\right)}$, where $\left(f_{\varphi} \mid \tau\right)=: \int_{\Omega} f_{\varphi}(x) \tau(x) d x$.

4. We say that an asymptotic function $\widehat{f}_{\varphi} \in \widehat{\mathcal{E}(\Omega)^{\mathcal{D}_{0}}}$ is weakly equal to zero in $\widehat{\mathcal{E}(\Omega)^{\mathcal{D}_{0}}}$, in symbol $\widehat{f}_{\varphi} \cong 0$, if $\left(\widehat{f}_{\varphi} \mid \tau\right)=0$ for all $\tau \in \mathcal{D}(\Omega)$. We say that $\widehat{f_{\varphi}}, \widehat{g_{\varphi}} \in \widehat{\mathcal{E}(\Omega)^{\mathcal{D}_{0}}}$ are weakly equal, in symbol $\widehat{f}_{\varphi} \cong{\widehat{g_{\varphi}}}_{\varphi}$, if $\left(\widehat{f}_{\varphi} \mid \tau\right)=\left(\widehat{g}_{\varphi} \mid \tau\right)$ in $\widehat{\mathbb{C}^{\mathcal{D}_{0}}}$ for all $\tau \in \mathcal{D}(\Omega)$.

5. We say that an asymptotic function $\widehat{f}_{\varphi} \in \widehat{\mathcal{E}(\Omega)^{\mathcal{D}_{0}}}$ is weakly infinitesimal (or, associated to zero), in symbol $\widehat{f}_{\varphi} \approx 0$, if $\left(\widehat{f}_{\varphi} \mid \tau\right) \approx 0$ for all $\tau \in \mathcal{D}(\Omega)$, where the latter $\approx$ is the infinitesimal relation on $\widehat{\mathbb{C}}^{\mathcal{D}_{0}}$ (Definition 4.5 ). We say that $\widehat{f}_{\varphi}, \widehat{g}_{\varphi} \in \widehat{\mathcal{E}(\Omega)^{\mathcal{D}_{0}}}$ are weakly infinitesimal (or, associated), in symbol $\widehat{f_{\varphi}} \approx \widehat{g_{\varphi}}$, if $\left(\widehat{f_{\varphi}} \mid \tau\right) \approx\left(\widehat{g_{\varphi}} \mid \tau\right)$ for all $\tau \in \mathcal{D}(\Omega)$, where in the latter formula $\approx$ stands for the infinitesimal relation in $\widehat{\mathbb{C}^{\mathcal{D}_{0}}}$. 
6. Let $\widehat{f}_{\varphi} \in \widehat{\mathcal{E}(\Omega)^{\mathcal{D}_{0}}}$ and let $\mathcal{O}$ be an open subset of $\Omega$. We define the restriction $\widehat{f}_{\varphi} \uparrow \mathcal{O} \in \widehat{\mathcal{E}(\mathcal{O})^{\mathcal{D}}}$ of $\widehat{f}_{\varphi}$ to $\mathcal{O}$ by $\widehat{f}_{\varphi} \uparrow \mathcal{O}=\widehat{f_{\varphi} \uparrow \mathcal{O}}$, where $f_{\varphi} \uparrow \mathcal{O}$ is the usual restriction of $f_{\varphi}$ to $\mathcal{O}$. The support $\operatorname{supp}\left(\widehat{f}_{\varphi}\right)$ of $\widehat{f}_{\varphi}$ is the complement to $\Omega$ of the largest open subset $G$ of $\Omega$ such that $\widehat{f}_{\varphi} \uparrow G=0$ in $\widehat{\mathcal{E}(G)^{\mathcal{D}}}$.

7. Let $\Omega, \Omega^{\prime} \in \mathcal{T}^{d}$ and $\psi \in \operatorname{Diff}\left(\Omega^{\prime}, \Omega\right)$ be a diffeomorphism. For every $\widehat{f}_{\varphi} \in \widehat{\mathcal{E}(\Omega)^{\mathcal{D}_{0}}}$ we define the composition (or, change of variables) $\widehat{f}_{\varphi} \circ \psi \in \widehat{\mathcal{E}\left(\Omega^{\prime}\right)^{\mathcal{D}_{0}}}$ by the formula $\widehat{f}_{\varphi} \circ \psi=\widehat{f_{\varphi} \circ \psi}$, where $f_{\varphi} \circ \psi$ stands for the usual composition between $f_{\varphi}$ and $\psi$.

It is clear that $\mathcal{M}\left(\mathcal{E}(\Omega)^{\mathcal{D}_{0}}\right)$ is a differential ring and $\mathcal{N}\left(\mathcal{E}(\Omega)^{\mathcal{D}_{0}}\right)$ is a differential ideal in $\mathcal{M}\left(\mathcal{E}(\Omega)^{\mathcal{D}_{0}}\right)$. Thus $\widehat{\mathcal{E}(\Omega)^{\mathcal{D}_{0}}}$ is a differential ring. We leave to the reader to verify that the product $\widehat{A_{\varphi}} \widehat{f}_{\varphi}$ is correctly defined. Thus we have the following result:

4.10 Theorem (Differential Algebra). $\widehat{\mathcal{E}(\Omega)^{\mathcal{D}}}$ is a differential algebra over the field $\widehat{\mathbb{C D}^{\mathcal{D}_{0}}}$.

\section{A Solution to the Problem of Multiplication of Schwartz Distributions}

In this section we construct a canonical embedding $E_{\Omega}$ of the space $\mathcal{D}^{\prime}(\Omega)$ of Schwartz distributions into the algebra of asymptotic functions $\widehat{\mathcal{E}(\Omega)^{\mathcal{D}_{0}}}$. Thus $\widehat{\mathcal{E}(\Omega)^{\mathcal{D}_{0}}}$ becomes a full algebra of generalized functions of Colombeau type (see the Introduction).

The algebra of asymptotic functions $\widehat{\mathcal{E}(\Omega)^{\mathcal{D}}}$ supplied with the embedding $E_{\Omega}$ offers a solution to the problem of the multiplication of Schwartz distributions similar to but different from Colombeau's solution (Colombeau [6]).

5.1 Definition (Embeddings). Let $\Omega$ be an open set of $\mathbb{R}^{d}$.

1. The standard embedding $\sigma_{\Omega}: \mathcal{E}(\Omega) \rightarrow \widehat{\mathcal{E}(\Omega)^{\mathcal{D}}}$ is defined by the constant nets, i.e. by the formula $\sigma_{\Omega}(f)=\widehat{f}$.

2. The distributional embedding $E_{\Omega}: \mathcal{D}^{\prime}(\Omega) \rightarrow \widehat{\mathcal{E}(\Omega)^{\mathcal{D}}}$ is defined by the formula $E_{\Omega}(T)=\widehat{T \circledast \varphi}$, where $T \circledast \varphi$ is the $\varphi$-regularization of $T \in \mathcal{D}^{\prime}(\Omega)$ (\# 5 in Examples 3.2). 
3. The classical function embedding $E_{\Omega} \circ L_{\Omega}: \mathcal{L}_{l o c}(\Omega) \rightarrow \widehat{\mathcal{E}(\Omega)^{\mathcal{D}}}$ is defined by the formula $\left(E_{\Omega} \circ L_{\Omega}\right)(f)=\widehat{f \circledast \varphi}$, where $f \circledast \varphi$ is the $\varphi$-regularization of $f \in \mathcal{L}_{\text {loc }}(\Omega)(\#[$ in Examples 3.2) .

5.2 Lemma (Correctness). The constant nets are moderate in the sense that $f \in$ $\mathcal{E}(\Omega)$ implies $(f) \in \mathcal{M}\left(\mathcal{E}(\Omega)^{\mathcal{D}_{0}}\right)$ (Section 4$)$. Similarly the $\varphi$-regularization of a Schwartz distribution (\#5 in Examples [.2) is also a moderate net, i.e. $T \in \mathcal{D}^{\prime}(\Omega)$ implies $(T \circledast \varphi) \in \mathcal{M}\left(\mathcal{E}(\Omega)^{\mathcal{D}_{0}}\right)$.

Proof. It is clear that the constant nets are moderate. To show the moderateness of $(T \circledast \varphi)$, suppose that $K \Subset \Omega$ and $\alpha \in \mathbb{N}_{0}$. By Lemma 3.4 there exist $m, n \in \mathbb{N}_{0}$ such that $\mathcal{D}_{n} \subseteq\left\{\varphi \in \mathcal{D}_{0}:(\forall x \in K)\left|\partial^{\alpha}(T \circledast \varphi)(x)\right| \leq\left(R_{\varphi}\right)^{-m}\right\}$ implying $\left\{\varphi \in \mathcal{D}_{0}\right.$ : $\left.\sup _{x \in K}\left|\partial^{\alpha}(T \circledast \varphi)(x)\right| \leq\left(R_{\varphi}\right)^{-m}\right\} \in \mathcal{U}$, as required.

Notice that the embedding $E_{\Omega}$ is canonical in the sense that it is uniquely defined in terms already used in the definition of the family $\left\{\widehat{\mathcal{E}(\Omega)^{\mathcal{D}_{0}}}\right\}_{\Omega \in \mathcal{T}^{d}}$ (Definition 4.9).

5.3 Theorem (Properties of Embedding). Let $\Omega$ be an open set of $\mathbb{R}^{d}$. Then:

(i) We have $\left(E_{\Omega} \circ L_{\Omega}\right)(f)=\sigma_{\Omega}(f)$ for all $f \in \mathcal{E}(\Omega)$. This can be summarized in the following commutative diagram:

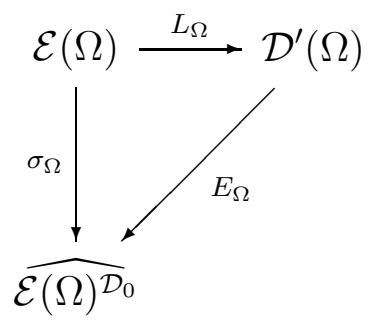

Consequently, $\mathcal{E}(\Omega)$ and $\left(E_{\Omega} \circ L_{\Omega}\right)[\mathcal{E}(\Omega)]$ are isomorphic differential algebras over $\mathbb{C}$. Also, $E_{\Omega} \circ L_{\Omega}=\sigma_{\Omega}$ preserves the pairing between $\mathcal{E}(\Omega)$ and $\mathcal{D}(\Omega)$ in the sense that

$$
\int_{\Omega} f(x) \tau(x) d x=\left(\sigma_{\Omega}(f) \mid \tau\right)=\left(\left(E_{\Omega} \circ L_{\Omega}\right)(f) \mid \tau\right),
$$

for all $f \in \mathcal{E}(\Omega)$ and all $\tau \in \mathcal{D}(\Omega)$. Consequently, $E_{\Omega} \circ L_{\Omega}=\sigma_{\Omega}$ is injective.

(ii) $E_{\Omega}$ is $\mathbb{C}$-linear and it preserves the partial differentiation of any order in $\mathcal{D}^{\prime}(\Omega)$. Also, $E_{\Omega}$ preserves the pairing between $\mathcal{D}^{\prime}(\Omega)$ and $\mathcal{D}(\Omega)$ in the sense that $(T \mid \tau)=\left(E_{\Omega}(T) \mid \tau\right)$ for all $T \in \mathcal{D}^{\prime}(\Omega)$ and all $\tau \in \mathcal{D}(\Omega)$. Consequently, $E_{\Omega}$ is injective. 
(iii) $E_{\Omega} \circ L_{\Omega}$ is $\mathbb{C}$-linear. Also, $E_{\Omega} \circ L_{\Omega}$ preserves the pairing between $\mathcal{L}_{\text {loc }}(\Omega)$ and $\mathcal{D}(\Omega)$ in the sense that

$$
\int_{\Omega} f(x) \tau(x) d x=\left(\left(E_{\Omega} \circ L_{\Omega}\right)(f) \mid \tau\right),
$$

for all $f \in \mathcal{L}_{\text {loc }}(\Omega)$ and all $\tau \in \mathcal{D}(\Omega)$. Consequently, $E_{\Omega} \circ L_{\Omega}$ is injective.

(iv) Each of the above embeddings: $\sigma_{\Omega}, E_{\Omega}$ and $E_{\Omega} \circ L_{\Omega}$, is sheaf preserving in the sense that it preserves the restriction to an open subset.

We summarize all of the above in $\mathcal{E}(\Omega) \subset \mathcal{L}_{\text {loc }}(\Omega) \subset \mathcal{D}^{\prime}(\Omega) \subset \widehat{\mathcal{E}(\Omega)^{\mathcal{D}_{0}}}$, where: (a) $\mathcal{E}(\Omega)$ is a differential subalgebra of $\widehat{\mathcal{E}(\Omega)^{\mathcal{D}_{0}}}$ over $\mathbb{C}$; (b) $\mathcal{L}_{\text {loc }}(\Omega)$ is a vector subspace of $\widehat{\mathcal{E}(\Omega)^{\mathcal{D}_{0}}}$ over $\mathbb{C}$ and $(c) \mathcal{D}^{\prime}(\Omega)$ is a differential vector subspace of $\widehat{\mathcal{E}(\Omega)^{\mathcal{D}}}$ over $\mathbb{C}$. We shall often write simply $T$ instead of the more precise $E_{\Omega}(T)$ for a Schwartz distribution in the framework of $\widehat{\mathcal{E}(\Omega)^{\mathcal{D}_{0}}}$.

Proof. (i) Suppose that $K \Subset \Omega, \alpha \in \mathbb{N}_{0}^{d}$ and $p \in \mathbb{N}$ (are chosen arbitrarily). By Lemma 3.5 there exist $n \in \mathbb{N}_{0}$ such that

$$
\mathcal{D}_{n} \subseteq\left\{\varphi \in \mathcal{D}_{0}: \sup _{x \in K}\left|\partial^{\alpha}(f \circledast \varphi)(x)-\partial^{\alpha} f(x)\right| \leq\left(R_{\varphi}\right)^{p}\right\} .
$$

Thus $\left\{\varphi \in \mathcal{D}_{0}: \sup _{x \in K}\left|\partial^{\alpha}(f \circledast \varphi)(x)-\partial^{\alpha} f(x)\right| \leq\left(R_{\varphi}\right)^{p}\right\} \in \mathcal{U}$. The latter means that the net $(f \circledast \varphi-f)$ is negligible (Definition 4.9) thus $\left(E_{\Omega} \circ L_{\Omega}\right)(f)=\widehat{f \circledast \varphi}=\widehat{f}=$ $\sigma_{\Omega}(f)$ as required. Consequently, we have $\left(E_{\Omega} \circ L_{\Omega}\right)[\mathcal{E}(\Omega)]=\sigma_{\Omega}[\mathcal{E}(\Omega)]$. Thus $\mathcal{E}(\Omega)$ and $\left(E_{\Omega} \circ L_{\Omega}\right)[\mathcal{E}(\Omega)]$ are isomorphic differential algebras because $\mathcal{E}(\Omega)$ and $\sigma_{\Omega}[\mathcal{E}(\Omega)]$ are (obviously) isomorphic differential algebras. Also, $E_{\Omega} \circ L_{\Omega}$ preserves the pairing because $\sigma_{\Omega}$ preserves (obviously) the pairing.

(ii) $\Sigma_{\Omega}$ is $\mathbb{C}$-linear because the mapping $T \rightarrow T \circledast \varphi$ is $\mathbb{C}$-linear. To show the preservation of partial differentiation we have to show that for every multi-index $\beta \in \mathbb{N}_{0}^{d}$ the net $\left(\partial^{\beta} T \circledast \varphi-\partial^{\beta}(T \circledast \varphi)\right)$ is negligible (Definition 4.9). This follows easily from Lemma 3.3 similarly to (i) above. To show that $E_{\Omega}$ preserves the pairing, we have to show that for any test function $\tau$ the net $A_{\varphi}=:(T \circledast \varphi \mid \tau)-(T \mid \tau)$ is negligible (Definition 4.1). The latter follows easily from Lemma 3.6.

(iii) $\left(E_{\Omega} \circ L_{\Omega}\right)$ is $\mathbb{C}$-linear because the mapping $f \rightarrow f \circledast \varphi$ is $\mathbb{C}$-linear. The preserving of pairing follows from (ii) in the particular case $T=T_{f}$.

(iv) The preserving of the restriction on an open subset follows easily from the definition and we leave the details to the reader. 
We should mention that if $f \in \mathcal{E}(\Omega)$ and $T \in \mathcal{D}^{\prime}(\Omega)$, then $E_{\Omega}(f) E_{\Omega}(T)=E_{\Omega}(f T)$ is false in general. That means that the multiplication in the algebra in $\widehat{\mathcal{E}(\Omega)^{\mathcal{D}_{0}}}$ does not reproduce the Schwartz multiplication in $\mathcal{D}^{\prime}(\Omega)$ (multiplication by duality). Similarly, let $\mathcal{C}(\Omega)$ denote the class of continuos functions from $\Omega$ to $\mathbb{C}$. If $g, h \in$ $\mathcal{C}(\Omega)$, then $E_{\Omega}(g) E_{\Omega}(h)=E_{\Omega}(g h)$ is also false in general. That means that the multiplication in the algebra in $\widehat{\mathcal{E}(\Omega)^{\mathcal{D}}}$ does not reproduce the usual multiplication in $\mathcal{C}(\Omega)$. Of course, all these are inevitable in view of the Schwartz impossibility results (Schwartz [34]). For a discussion we refer to (Colombeau [10], p. 8). Instead, we have a somewhat weaker result.

5.4 Theorem (Weak Preservation). Let $T \in \mathcal{D}^{\prime}(\Omega), f \in \mathcal{E}(\Omega)$ and $g, h \in \mathcal{C}(\Omega)$. Then:

(i) $E_{\Omega}(f) E_{\Omega}(T) \cong E_{\Omega}(f T)$ (Definition [4.9, \#乐), i.e. $\left(E_{\Omega}(f) E_{\Omega}(T) \mid \tau\right)=\left(E_{\Omega}(f T) \mid \tau\right)$ for all $\tau \in \mathcal{D}(\Omega)$.

(ii) $E_{\Omega}(g) E_{\Omega}(h) \approx E_{\Omega}(g h)$ (Definition 4.9, \#55), i.e. $\left(E_{\Omega}(g) E_{\Omega}(h) \mid \tau\right) \approx\left(E_{\Omega}(g h) \mid \tau\right)$ for all $\tau \in \mathcal{D}(\Omega)$, where $\approx$ in the latter formula stands for the infinitesimal relation in the field $\widehat{\mathbb{C}^{\mathcal{D}_{0}}}$.

Proof. (i) We denote $f_{\varphi, \tau}:=(f(T \circledast \varphi) \mid \tau)=(T \circledast \varphi \mid f \tau)$ and calculate

$\left(E_{\Omega}(f) E_{\Omega}(T) \mid \tau\right)=(\widehat{f} \widehat{T \circledast \varphi} \mid \tau)=(f \widehat{(T \circledast \varphi)} \mid \tau)=\widehat{f_{\varphi, \tau}}=(\widehat{T \circledast \varphi} \mid f \tau)=$ $(T \mid f \tau)=(f T \mid \tau)=\left(E_{\Omega}(f T) \mid \tau\right)$ as required.

(ii) This follows from the fact that for each $n \in \mathbb{N}$ and $K \Subset \Omega$ we have $\sup _{x \in K} \mid(g \circledast$ $\varphi-g)(x) h(x) \mid<1 / n$ and $\sup _{x \in K}|(g \circledast \varphi)(x)(h \circledast \varphi-h)(x)|<1 / n$ a.e. in $\mathcal{D}_{0}$ (Definition 2.5) which can be seen by elementary observation.

Let $\Omega, \Omega^{\prime} \in \mathcal{T}^{d}$ and $\psi \in \operatorname{Diff}\left(\Omega^{\prime}, \Omega\right)$. Then $E_{\Omega}(T) \circ \psi=E_{\Omega^{\prime}}(T \circ \psi)$ does not generally hold in $\widehat{\mathcal{E}(\Omega)^{\mathcal{D}_{0}}}$. That means that the family of algebras $\left\{\widehat{\mathcal{E}(\Omega)^{\mathcal{D}_{0}}}\right\}_{\Omega \in \mathcal{T}^{d}}$ is not diffeomorphism invariant (see the Introduction). Here $T \circ \psi$ stands for the composition in the sense of the distribution theory (Vladimirov [40]). Instead, we have the following weaker result.

5.5 Theorem (Diffeomorphisms). $E_{\Omega}$ weakly preserves the composition with diffeomorphisms in the sense that for every $\Omega, \Omega^{\prime} \in \mathcal{T}^{d}$, every $T \in \mathcal{D}^{\prime}(\Omega)$ and every $\psi \in$ $\operatorname{Diff}\left(\Omega^{\prime}, \Omega\right)$ we have $E_{\Omega}(T) \circ \psi \cong E_{\Omega^{\prime}}(T \circ \psi)$, i.e. $\left(E_{\Omega}(T) \circ \psi \mid \tau\right)=\left(E_{\Omega^{\prime}}(T \circ \psi) \mid \tau\right)$ for all test functions $\tau \in \mathcal{D}\left(\Omega^{\prime}\right)$.

Proof. The proof is analogous to the proof of part (i) of Theorem 5.4 and we leave the details to the reader. 
5.6 Examples. 1. Let $\delta \in \mathcal{D}^{\prime}\left(\mathbb{R}^{d}\right)$ be the Dirac delta function (delta distribution) on $\mathbb{R}^{d}$. For its $\varphi$-regularization (\#5 in Examples 3.2 ) we have $\delta_{\varphi}=\delta \circledast \varphi=$ $\delta \star \varphi=\varphi$. Thus $E_{\mathbb{R}^{d}}(\delta)=\widehat{\varphi}$. Similarly, $E_{\mathbb{R}^{d}}\left(\partial^{\alpha} \delta\right)=\widehat{\partial^{\alpha} \varphi}$.

2. We have $\left(E_{\mathbb{R}^{d}}(\delta)\right)^{n}=(\widehat{\varphi})^{n}=\widehat{\varphi^{n}}, n=1,2, \ldots$ We express this result simply as $\delta^{n}=\widehat{\varphi^{n}}$. Recall that the powers $\delta^{n}$ are meaningless within $\mathcal{D}^{\prime}\left(\mathbb{R}^{d}\right)$ for $n \geq 2$.

3. Let $H(x)$ be the Heaviside step function on $\mathbb{R}$. For its $\varphi$-regularization (\#6 in Examples [3.2) we have $H_{\varphi}=(H \circledast \varphi)$. Let $K \Subset \mathbb{R}$. We observe that for every $x \in K$ we have $H_{\varphi}(x)=(H \star \varphi)(x)=\int_{-\infty}^{x} \varphi(t) d t$ a.e. in $\mathcal{D}_{0}$ (Definition 2.5). Thus $E_{\mathbb{R}}(H)=\int_{-\infty}^{x} \widehat{\varphi(t)} d t$. We express this result simply as $H(x)=\int_{-\infty}^{x} \widehat{\varphi(t)} d t$. Since the embedding $E_{\mathbb{R}}$ preserves the differentiation, we have $H^{\prime}=\delta$.

4. We have $E_{\mathbb{R}}(H) E_{\mathbb{R}}(\delta)=\widehat{\varphi}\left(\int_{-\infty}^{x} \widehat{\varphi(t)} d t\right)={\widehat{\varphi H_{\varphi}}}$. We express this result simply

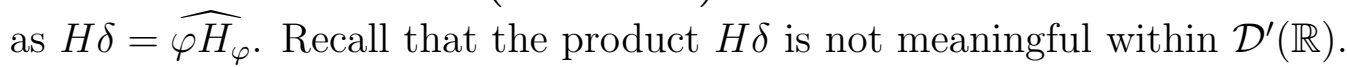

5. We have $\left(E_{\mathbb{R}}(H)\right)^{n}=\left(\int_{-\infty}^{x} \widehat{\varphi(t)} d t\right)^{n}=\widehat{\left(H_{\varphi}\right)^{n}}$ which we write simply as $H^{n}=$ $\widehat{\left(H_{\varphi}\right)^{n}}$. Since $\widehat{\mathcal{E}\left(\mathbb{R}^{\mathcal{D}_{0}}\right.}$ is a differential algebra, we can apply the chain rule: $\left(H^{n}\right)^{\prime}=n H^{n-1} \delta$ which also is meaningless in $\mathcal{D}^{\prime}(\mathbb{R})$ for $n \geq 2$.

6. Notice that $H^{n} \neq H, n=2,3, \ldots$ in $\widehat{\mathcal{E}\left(\widehat{\mathbb{R}}^{\mathcal{D}_{0}}\right.}$. Actually $H^{n}=H, n=2,3, \ldots$, fail in any differential algebra. Indeed, $H^{2}=H$ implies $2 H \delta=\delta$ while $H^{3}=H$ implies $3 H \delta=\delta$ thus $2=3$, a contradiction. For a discussion we refer to (Grosser, Farkas, Kunzinger \& Steinbauer [14], Example (1.1.1)).

\section{Distributional Non-Standard Model}

The distributional non-standard model presented in this section is especially designed for the purpose of the non-linear theory of generalized functions (Colombeau theory). It is a $\mathfrak{c}^{+}$-saturated ultrapower non-standard model with the set of individuals $\mathbb{R}$ based on the $\mathcal{D}_{0}$-nets (Definition 3.1). Here $\mathfrak{c}=\operatorname{card}(\mathbb{R})$ and $\mathfrak{c}^{+}$stands for the successor of $\mathfrak{c}$. The connection of the theory of asymptotic numbers and functions (Section 4) with non-standard analysis will be discussed in the next section. We should mention that a similar ultrapower non-standard model (with the same index set and different ultrafilter) was used in Guy Berger's thesis [1] for studying delta-like solutions of Hopf's equation. 
For readers who are familiar with non-standard analysis this section is a short review of the ultra-power approach to non-standard analysis introduced by W. A. J. Luxemburg [22] almost 40 years ago (see also Stroyan \& Luxemburg [35]). For the reader without background in non-standard analysis, this section offers a short introduction to the subject. For additional reading, we refer to Davis [12], Lindstrøm [21] and Chapter 2 in Capiński \& Cutland [4].

6.1 Definition (Distributional Non-Standard Model). $\quad 1$. Let $S$ be an infinite set. The superstructure on $S$ is defined by $V(S)=: \bigcup_{n=0}^{\infty} V_{n}(S)$, where $V_{0}(S)=S$ and $V_{n+1}(S)=V_{n}(S) \cup \mathcal{P}\left(V_{n}(S)\right)$. The level $\lambda(A)$ of $A \in V(S)$ is defined by the formula $\lambda(A)=: \min \left\{n \in \mathbb{N}_{0}: A \in V_{n}(S)\right\}$. The superstructure $V(S)$ is transitive in the sense that $V(S) \backslash S \subset \mathcal{P}(V(S))$. Thus $V(S) \backslash S$ is a Boolean algebra. The members $s$ of $S$ are called individuals of the superstructure $V(S)$.

2. Let $S=\mathbb{R}$. We observe that $V(\mathbb{R})$ contains all objects in standard analysis: all ordered pairs of real numbers thus the set of complex numbers $\mathbb{C}$, Cartesian products of subsets of $\mathbb{R}$ and of $\mathbb{C}$ thus all relations on $\mathbb{R}$ and on $\mathbb{C}$, all binary algebraic operations on $\mathbb{R}$ and on $\mathbb{C}$, all real and complex functions, all sets of functions, etc.

3. Let $\mathbb{R}^{\mathcal{D}_{0}}$ be the set of all $\mathcal{D}_{0}$-nets in $\mathbb{R}$ (Definition 3.1 ). The set ${ }^{*} \mathbb{R}$ of nonstandard real numbers is defined as follows:

(a) We define the equivalence relation $\sim_{\mathcal{U}}$ on $\mathbb{R}^{\mathcal{D}_{0}}$ by $\left(A_{\varphi}\right) \sim_{\mathcal{U}}\left(B_{\varphi}\right)$ if $A_{\varphi}=B_{\varphi}$ a.e. or, equivalently, if $\left\{\varphi \in \mathcal{D}_{0}: A_{\varphi}=B_{\varphi}\right\} \in \mathcal{U}$ (Definition 2.5).

(b) The equivalence classes in ${ }^{*} \mathbb{R}=\mathbb{R}^{\mathcal{D}_{0}} / \sim_{\mathcal{U}}$ are called non-standard real numbers. We denote by $\left\langle A_{\varphi}\right\rangle \in{ }^{*} \mathbb{R}$ the equivalence class of the net $\left(A_{\varphi}\right) \in \mathbb{R}^{\mathcal{D}_{0}}$. The ring operations in ${ }^{*} \mathbb{R}$ are inherited from the ring $\mathbb{R}^{\mathcal{D}_{0}}$. The order in ${ }^{*} \mathbb{R}$ is defined by $\left\langle A_{\varphi}\right\rangle>0$ if $A_{\varphi}>0$ a.e., i.e. if $\left\{\varphi \in \mathcal{D}_{0}\right.$ : $\left.A_{\varphi}>0\right\} \in \mathcal{U}$.

(c) We define the canonical embedding $\mathbb{R} \hookrightarrow^{*} \mathbb{R}$ by the constant nets, i.e. by $A \rightarrow\left\langle A_{\varphi}\right\rangle$, where $A_{\varphi}=A$ for all $\varphi \in \mathcal{D}_{0}$. We shall write simply $\mathbb{R} \subseteq{ }^{*} \mathbb{R}$ instead of $\mathbb{R} \hookrightarrow{ }^{*} \mathbb{R}$. Also if $\left(A_{\varphi}\right)$ is a constant net, we shall write simply $\langle A\rangle$ instead of $\left\langle A_{\varphi}\right\rangle$.

4. Let $S={ }^{*} \mathbb{R}$. The superstructure $V\left({ }^{*} \mathbb{R}\right)$ contains all objects in non-standard analysis: ordered pairs of non-standard real numbers thus the set of nonstandard complex numbers ${ }^{*} \mathbb{C}$, all Cartesian products of subsets of * $\mathbb{R}$ and of ${ }^{*} \mathbb{C}$ thus all relations on ${ }^{*} \mathbb{R}$ and on ${ }^{*} \mathbb{C}$, all binary algebraic operations on ${ }^{*} \mathbb{R}$ and on ${ }^{*} \mathbb{C}$, all non-standard functions, all sets of non-standard functions, etc. 
5. Let $V(\mathbb{R})^{\mathcal{D}_{0}}$ stand for the set of all $\mathcal{D}_{0}$-nets in $V(\mathbb{R})$ (Definition [3.1). A net $\left(A_{\varphi}\right)$ in $V(\mathbb{R})^{\mathcal{D}_{0}}$ is called tame if $\left(\exists n \in \mathbb{N}_{0}\right)\left(\forall \varphi \in \mathcal{D}_{0}\right)\left(A_{\varphi} \in V_{n}(\mathbb{R})\right)$. If $\left(A_{\varphi}\right)$ is a tame net in $V(\mathbb{R})^{\mathcal{D}_{0}}$ its level $\lambda\left(\left(A_{\varphi}\right)\right)$ is defined (uniquely) as the number $n \in \mathbb{N}_{0}$ such that $\left\{\varphi \in \mathcal{D}_{0}: \lambda\left(A_{\varphi}\right)=n\right\} \in \mathcal{U}$, where $\lambda\left(A_{\varphi}\right)$ is the level of $A_{\varphi}$ in $V(\mathbb{R})$ (see \#1 above).

6. For every tame net $\left(A_{\varphi}\right)$ in $V(\mathbb{R})^{\mathcal{D}_{0}}$ we define $\left\langle A_{\varphi}\right\rangle \in V\left({ }^{*} \mathbb{R}\right)$ inductively on the level of the nets: If $\lambda\left(\left(A_{\varphi}\right)\right)=0$, then $\left\langle A_{\varphi}\right\rangle$ is defined in \#3 above. Suppose $\left\langle A_{\varphi}\right\rangle$ is already defined for all tame nets $\left(A_{\varphi}\right)$ in $V(\mathbb{R})^{\mathcal{D}_{0}}$ with $\lambda\left(\left(A_{\varphi}\right)\right)<$ $n$. If $\left(B_{\varphi}\right) \in V(\mathbb{R})^{\mathcal{D}_{0}}$ is a tame net with $\lambda\left(\left(B_{\varphi}\right)\right)=n$, we let $\left\langle B_{\varphi}\right\rangle=$ : $\left\{\left(A_{\varphi}\right) \in V(\mathbb{R})^{\mathcal{D}_{0}}: \lambda\left(\left(A_{\varphi}\right)\right)<n \& A_{\varphi} \in B_{\varphi}\right.$ a.e. $\}$, where, as before, $A_{\varphi} \in B_{\varphi}$ a.e. means $\left\{\varphi \in \mathcal{D}_{0}: A_{\varphi} \in B_{\varphi}\right\} \in \mathcal{U}$ (Definition 2.5). Let $\left(A_{\varphi}\right)$ be a constant net in $V(\mathbb{R})^{\mathcal{D}_{0}}$, i.e. $A_{\varphi}=A$ for all $\varphi \in \mathcal{D}_{0}$ and some $A \in V(\mathbb{R})$. In the case of constant nets we shall write simply $\langle A\rangle$ instead of $\left\langle A_{\varphi}\right\rangle$.

7. An element $\mathcal{A}$ of $V\left({ }^{*} \mathbb{R}\right)$ is called internal if $\mathcal{A}=\left\langle A_{\varphi}\right\rangle$ for some tame net $\left(A_{\varphi}\right) \in V(\mathbb{R})^{\mathcal{D}_{0}}$. We denote by ${ }^{*} V(\mathbb{R})$ the set of the internal elements of $V\left({ }^{*} \mathbb{R}\right)$ (including the non-standard reals in ${ }^{*} \mathbb{R}$ ). The elements of ${ }^{*} V(\mathbb{R}) \backslash{ }^{*} \mathbb{R}$ are called internal sets. The internal sets of the form $\langle A\rangle$, where $A \in V(\mathbb{R})$ (i.e. generated by constant nets), are called internal standard (or simply, standard). The elements of $V\left({ }^{*} \mathbb{R}\right) \backslash{ }^{*} V(\mathbb{R})$ are called external sets.

8. We define the extension mapping $*: V(\mathbb{R}) \rightarrow V\left({ }^{*} \mathbb{R}\right)$ by ${ }^{*} A=\langle A\rangle$. Notice that the range $\operatorname{ran}(*)$ of the extension mapping $*$ consists exactly of the internal standard elements of $V\left({ }^{*} \mathbb{R}\right)$. The terminology extension mapping for $*$ is due to the following result: Let $S \in V(\mathbb{R}) \backslash \mathbb{R}$. Then $S \subseteq{ }^{*} S$ and the equality occurs iff $S$ is a finite set.

9. It can be shown that $\mathcal{A}$ is internal iff $\mathcal{A} \in{ }^{*} A$ for some $A \in V(\mathbb{R})$. It can be shown as well that an element $A \in V(\mathbb{R})$ is internal iff $A \in \mathbb{R}$ or $A$ is a finite set (notice that $V(\mathbb{R}) \subseteq V\left({ }^{*} \mathbb{R}\right)$ since $\mathbb{R} \subseteq{ }^{*} \mathbb{R}$ ). The infinite sets in $V(\mathbb{R}) \backslash \mathbb{R}$ are called external standard sets. For example, the familiar $\mathbb{N}, \mathbb{N}, \mathbb{Z}, \mathbb{Q}, \mathbb{R}, \mathbb{C}$ are all external standard sets.

10. A point $\zeta \in{ }^{*} \mathbb{C}^{d}$ is called infinitesimal if $\|\zeta\|<1 / n$ for all $n \in \mathbb{N}$. Also, $\zeta \in{ }^{*} \mathbb{C}^{d}$ is called finite if $\|\zeta\|<n$ for some $n \in \mathbb{N}$. Similarly, $\zeta \in{ }^{*} \mathbb{C}^{d}$ is called infinitely large if $n<\|\zeta\|$ for all $n \in \mathbb{N}$. We denote by $\mathcal{I}\left({ }^{*} \mathbb{C}^{d}\right), \mathcal{F}\left({ }^{*} \mathbb{C}^{d}\right)$ and $\mathcal{L}\left({ }^{*} \mathbb{C}^{d}\right)$ the sets of the infinitesimal, finite and infinitely large points in ${ }^{*} \mathbb{C}^{d}$, respectively. We often write $\zeta \approx 0$ instead of $\zeta \in \mathcal{I}\left({ }^{*} \mathbb{C}^{d}\right)$ and $\zeta_{1} \approx \zeta_{2}$ instead of $\zeta_{1}-\zeta_{2} \in \mathcal{I}\left({ }^{*} \mathbb{C}^{d}\right)$. More generally, if $\mathcal{S} \subseteq{ }^{*} \mathbb{C}^{d}$, then $\mathcal{I}(\mathcal{S}), \mathcal{F}(\mathcal{S})$ and 
$\mathcal{L}(\mathcal{S})$ denote the sets of infinitesimal, finite and infinitely large points in $\mathcal{S}$, respectively.

11. We define the standard part mapping st $: \mathcal{F}\left({ }^{*} \mathbb{C}^{d}\right) \rightarrow \mathbb{C}^{d}$ by the formula $\operatorname{st}(\zeta) \approx \zeta$. We observe that st is a vector homomorphism from $\mathcal{F}\left({ }^{*} \mathbb{C}^{d}\right)$ onto $\mathbb{C}^{d}$. In particular, st $: \mathcal{F}\left({ }^{*} \mathbb{C}\right) \rightarrow \mathbb{C}$ is an order preserving ring homomorphism from $\mathcal{F}\left({ }^{*} \mathbb{C}\right)$ onto $\mathbb{C}$ (relative to the partial order in ${ }^{*} \mathbb{C}$ ).

12. We call $\rho \in{ }^{*} \mathbb{R}$, defined by $\rho=\left\langle R_{\varphi}\right\rangle$ (cf. (1)), the canonical infinitesimal in ${ }^{*} \mathbb{R}$. It is canonical because is defined uniquely in terms of the index set of the distributional non-standard model. It is a positive infinitesimal because $0<\rho<1 / n$ for all $n \in \mathbb{N}$ (Example 2.6).

13. Let $x \in \mathbb{R}^{d}$ and $X \subseteq \mathbb{R}^{d}$. The monads of $x$ and $X$ are defined by

$$
\begin{aligned}
& \mu(x)=\left\{x+d x: d x \in{ }^{*} \mathbb{R}^{d} \&\|d x\| \approx 0\right\}, \\
& \mu(X)=\left\{x+d x: x \in X \& d x \in{ }^{*} \mathbb{R}^{d} \&\|d x\| \approx 0\right\},
\end{aligned}
$$

respectively. Also, $\mu_{0}(x)=: \mu(x) \backslash\{x\}$ is the deleted monad of $x$.

6.2 Theorem (Extension Principle). ${ }^{*} \mathbb{R}$ is a proper extension of $\mathbb{R}$, i.e. $\mathbb{R} \varsubsetneqq{ }^{*} \mathbb{R}$. Consequently, $V(\mathbb{R}) \varsubsetneqq V(* \mathbb{R})$.

Proof. We observe that $\rho \in{ }^{*} \mathbb{R} \backslash \mathbb{R}(\# 12$ in Definition 6.1)

In what follows we assume a particular case of the continuum hypothesis in the form $\mathfrak{c}^{+}=2^{\mathfrak{c}}$.

6.3 Theorem (Saturation Principle). Our non-standard model $V\left({ }^{*} \mathbb{R}\right)$ is $\mathfrak{c}^{+}$-saturated in the sense that every family $\left(\mathcal{A}_{\gamma}\right)_{\gamma \in \Gamma}$ of internal sets in $V\left({ }^{*} \mathbb{R}\right)$ with the finite intersection property and $\operatorname{card}(\Gamma) \leq \mathfrak{c}$ has the non-empty intersection $\bigcap_{\gamma \in \Gamma} \mathcal{A}_{\gamma} \neq \varnothing$. Also $V\left({ }^{*} \mathbb{R}\right)$ is fully saturated in the sense that $V\left({ }^{*} \mathbb{R}\right)$ is card $\left({ }^{*} \mathbb{R}\right)$-saturated (cf. Chang \& Keisler [5], Chpter 5).

Proof. We refer the reader to the original proof in Chang \& Keisler [5] (for a presentation see also Lindstrøm [21]). We should mention that the property of the ultrafilter $\mathcal{U}$ to be $\mathfrak{c}^{+}$-good (\# 6 in Lemma 2.4) is involved in the proof of this theorem. To show that $V\left({ }^{*} \mathbb{R}\right)$ is fully saturated, we have to show that $\operatorname{card}\left({ }^{*} \mathbb{R}\right)=\mathfrak{c}^{+}$. Indeed, $\operatorname{card}\left({ }^{*} \mathbb{R}\right) \leq \operatorname{card}\left(\mathbb{R}^{\mathcal{D}_{0}}\right)=2^{\mathfrak{c}}$ follows from the definition of ${ }^{*} \mathbb{R}$ in the distributional model and $\operatorname{card}\left({ }^{*} \mathbb{R}\right) \geq 2^{\mathfrak{c}}$ follows from the fact that $V\left({ }^{*} \mathbb{R}\right)$ is $\mathfrak{c}^{+}$-saturated. 
6.4 Theorem (Order Completeness Principle). Let $\mathcal{A}$ be an internal non-empty bounded from above subset of ${ }^{*} \mathbb{R}$. Then $\sup (\mathcal{A})$ exists in ${ }^{*} \mathbb{R}$. Also, if $\sup (\mathcal{A})$ exists, then there exists a net $\left(\mathcal{A}_{\varphi}\right) \in \mathcal{P}(\mathbb{R})^{\mathcal{D}_{0}}$ (Examples [.2) such that $\mathcal{A}=\left\langle\mathcal{A}_{\varphi}\right\rangle$ and $\sup (\mathcal{A})=\left\langle\sup \left(\mathcal{A}_{\varphi}\right)\right\rangle$.

Proof. For the proof we refer to (Lindstrøm [21], p. 11).

6.5 Theorem (Spilling Principles). Let $\mathcal{A} \subseteq{ }^{*} \mathbb{R}$ be an internal set. Then:

(i) Overflow of $\mathcal{F}\left({ }^{*} \mathbb{R}\right)$ : If $\mathcal{A}$ contains arbitrarily large finite numbers, then $\mathcal{A}$ contains arbitrarily small infinitely large numbers.

(ii) Underflow of $\mathcal{F}\left({ }^{*} \mathbb{R}\right) \backslash \mathcal{I}\left({ }^{*} \mathbb{R}\right)$ : If $\mathcal{A}$ contains arbitrarily small finite noninfinitesimals, then $\mathcal{A}$ contains arbitrarily large infinitesimals.

(iii) Overflow of $\mathcal{I}\left({ }^{*} \mathbb{R}\right):$ If $\mathcal{A}$ contains arbitrarily large infinitesimals, then $\mathcal{A}$ contains arbitrarily small finite non-infinitesimals.

(iv) Underflow of $\mathcal{L}\left({ }^{*} \mathbb{R}\right)$ : If $\mathcal{A}$ contains arbitrarily small infinitely large numbers, then $\mathcal{A}$ contains arbitrarily large finite numbers.

Proof. For the proof we refer to Davis [12] or Lindstrøm [21].

The next result demonstrates the remarkable feature of non-standard analysis to reduce (and sometimes even to eliminate completely) the number of quantifiers compared with standard analysis.

6.6 Theorem (Usual Topology on $\mathbb{R}^{d}$ and Monads). Let $X \subseteq \mathbb{R}^{d}$ and $x \in \mathbb{R}^{d}$. Then: (a) $x$ is an interior point of $X$ iff $\mu(x) \subseteq{ }^{*} X$. Consequently, $X$ is open iff $\mu(X) \subseteq{ }^{*} X$. (b) $X$ is closed iff $\operatorname{st}\left({ }^{*} X\right)=X$, where st $: \mathcal{F}\left({ }^{*} \mathbb{R}^{d}\right) \rightarrow \mathbb{R}^{d}$ stands for the standard part mapping. (c) $x$ is an adherent point of $X$ (i.e. $x \in \bar{X}$ ) iff ${ }^{*} X \cap \mu(x) \neq \varnothing$. (d) $X$ is a cluster point of $X$ iff ${ }^{*} X \cap \mu_{0}(x) \neq \varnothing$. (e) $X$ is a bounded set iff ${ }^{*} X$ consists of finite points only. (f) $X$ is compact iff ${ }^{*} X \subseteq \mu(X)$.

Proof. We refer the reader to the original proofs in Robinson [31] (or, to a presentation in Salbany \& Todorov [33]).

The complete our survey on non-standard analysis we have to discuss two more important principles: the transfer principle and internal definition principle. The transfer principle is considered by many as the "heart and soul of non-standard analysis". The formulation of these two principles however requires a more precise choice of our formal language. The reader who do not have taste for mathematical logic might skip (or browse casually through) the rest of this section. 
6.7 Definition (Formal Language). Let $S$ be (as before) an infinite set and $V(S)$ be the superstructure on $S$. The formal language $\mathcal{L A N}(S)$ with set of individuals $S$ is constructed as follows:

1. The alphabet of $\mathcal{L} \mathcal{A N}(S)$ consists of the three mutually disjoint sets $\mathcal{A} \cup \mathcal{B} \cup$ $V(S)$, where

(a) $\mathcal{A}=\{=, \in, \neg, \wedge$ (and),$\vee($ or) $, \forall, \exists, \Rightarrow, \Leftrightarrow,(),[],\{\}\}$ is the (finite) set of symbols.

(b) $\mathcal{B}=\left\{x, y, z, X, Y, Z, x_{1}, x_{2}, \ldots\right\}$ is the countable set of variables.

The members of $\mathcal{A} \cup \mathcal{B} \cup V(S)$ are called letters. A word is any finite string of letters.

2. The vocabulary of the language $\mathcal{L} \mathcal{A N}(S)$ consists of: words, terms, predicates and propositions. They are defined recursively (by the length of the word), where all quantifiers in the predicates and propositions are bounded by elements in $V(S) \backslash S$. In other words, $\mathcal{L} \mathcal{A N}(S)$ allows only predicates (propositions) such as $\left(\forall x_{1} \in A\right) P\left(x_{1}, x_{2} \ldots x_{n}\right)$ or $\left(\forall x_{1} \in A\right)\left(\exists x_{2} \in B\right) P\left(x_{1}, x_{2} \ldots x_{n}\right)$, where $A, B \in V(S) \backslash S$. Here $P\left(x_{1}, x_{2} \ldots x_{n}\right)$ stands for a predicate in the free variables $x_{1}, \ldots x_{n} \in \mathcal{B}$. Notice that the language $\mathcal{L} \mathcal{A N}(S)$ disallows predicates (propositions) such as $\left(\forall x_{1}\right) P\left(x_{1}, x_{2} \ldots x_{n}\right)$ or $\left(\forall x_{1}\right)\left(\exists x_{2}\right) P\left(x_{1}, x_{2} \ldots x_{n}\right)$.

3. We supply the set of propositions in the language $\mathcal{L} \mathcal{A N}(S)$ with the usual semantics (true or false values) inherited from the Boolean structure of $V(S) \backslash$ $S$.

6.8 Examples. Here are our two basic examples:

1. Let $S=\mathbb{R}$. Then $\mathcal{L A N}(\mathbb{R})$ is the formal language of standard analysis.

2. Let $S={ }^{*} \mathbb{R}$. Then $\mathcal{L} \mathcal{A N}\left({ }^{*} \mathbb{R}\right)$ is the formal language of non-standard analysis.

For more details on the topic we refer to Davis [12], Lindstrøm [21] and Chapter 2 in Capiński \& Cutland [4]. We believe however that the reader can successfully proceed to the rest of this article without more specialized knowledge in logic.

6.9 Theorem (Transfer Principle). Let $P\left(x_{1}, \ldots, x_{q}\right)$ be a predicate in the language $\mathcal{L} \mathcal{A N}(\mathbb{R})$ and let $A_{n} \in V(\mathbb{R}), n=1,2, \ldots, q$. Let $P\left(A_{1}, \ldots, A_{q}\right)$ and $P\left({ }^{*} A_{1}, \ldots,{ }^{*} A_{q}\right)$ be the propositions in the languages $\mathcal{L} \mathcal{A N}(\mathbb{R})$ and $\mathcal{L} \mathcal{A N}\left({ }^{*} \mathbb{R}\right)$, respectively, obtained from $P\left(x_{1}, \ldots, x_{q}\right)$ by replacing all $x^{\prime} s$ by $A^{\prime}$ s or ${ }^{*} A^{\prime}$ s, respectively. Then $P\left(A_{1}, \ldots, A_{q}\right)$ and $P\left({ }^{*} A_{1}, \ldots,{ }^{*} A_{q}\right)$ are equivalent, i.e. $P\left(A_{1}, \ldots, A_{q}\right) \Leftrightarrow P\left({ }^{*} A_{1}, \ldots,{ }^{*} A_{q}\right)$. 
Proof. We refer the reader to Davis [12] (for a presentation, see also Lindstrøm [21]).

6.10 Corollary (Field Properties). ${ }^{*} \mathbb{R}$ is a non-archimedean real closed (thus, totally ordered) field. Consequently, ${ }^{*} \mathbb{C}$ is a non-archimedean algebraically closed field and we have the usual connection ${ }^{*} \mathbb{C}={ }^{*} \mathbb{R}(i)$, where $i=\sqrt{-1}$.

Proof. The field properties follow from the transfer principle. For example, the proposition $\left(\forall x \in{ }^{*} \mathbb{R}\right)\left[x \neq 0 \Rightarrow\left(\exists y \in{ }^{*} \mathbb{R}\right)(x y=1)\right]$ is true in $\mathcal{L} \mathcal{A} \mathcal{N}\left({ }^{*} \mathbb{R}\right)$ because $(\forall x \in \mathbb{R})[x \neq 0 \Rightarrow(\exists y \in \mathbb{R})(x y=1)]$ is true in $\mathcal{L} \mathcal{A N}(\mathbb{R})$ and similarly for the rest of the real closed field axioms and algebraically closed field axioms. Also both ${ }^{*} \mathbb{R}$ and ${ }^{*} \mathbb{C}$ are non-archimedean because they are proper extensions of $\mathbb{R}$ and $\mathbb{C}$, respectively.

6.11 Remark (An Alternative Proof). The above corollary can also be proved without transfer principle. We have to involve the nets in $\mathbb{R}^{\mathcal{D}_{0}}$ and $\mathbb{C}^{\mathcal{D}_{0}}$ and use the properties of the ultrafilter $\mathcal{U}$ listed in Lemma 2.4. This second proof is very similar to the proof of Theorem 4.2 .

6.12 Theorem (Internal Definition Principle). Let $\mathcal{A} \in{ }^{*} V(\mathbb{R}) \backslash{ }^{*} \mathbb{R}$ (be an internal set) and let $\mathcal{A}_{n} \in{ }^{*} V(\mathbb{R}), n=1, \ldots, q$ (be non-standard real numbers or internal sets). Let $P\left(x, x_{1}, \ldots, x_{q}\right)$ be a predicate in $q+1$ variables in the language $\mathcal{L} \mathcal{A N}(\mathbb{R})$ and let $P\left(x, \mathcal{A}_{1}, \ldots, \mathcal{A}_{q}\right)$ be the corresponding predicate in a single variable in the language $\mathcal{L} \mathcal{A N}\left({ }^{*} \mathbb{R}\right)$. Then the set $\mathcal{B}=:\left\{x \in \mathcal{A}: P\left(x, \mathcal{A}_{1}, \ldots, \mathcal{A}_{q}\right)\right\}$ is also internal.

Proof. We refer the reader to Davis [12].

6.13 Remark (Axiomatic Approach). The extension, saturation and transfer principles are theorems in the distributional model presented above. In one of the axiomatic approaches to non-standard analysis however these three principles are treated as axioms. For a discussion we refer to (Lindstrøm [21], pp. 81-83 and pp. 97-98).

\section{J.F. Colombeau's Theory of Generalized Func- tions and Non-Standard Analysis}

We show that the field of asymptotic numbers $\widehat{\mathbb{C}^{\mathcal{D}_{0}}}$ (Definition 4.1 ) is isomorphic to a particular Robinson field ${ }^{\rho} \mathbb{C}$ (Robinson [32]) of $\rho$-asymptotic numbers. We also prove that the algebra of asymptotic functions $\widehat{\mathcal{E}(\Omega)^{\mathcal{D}_{0}}}$ (Definition 4.9$)$ is isomorphic to a particular algebra of $\rho$-asymptotic functions ${ }^{\rho} \mathcal{E}(\Omega)$ introduced in (Oberguggenberger \& Todorov[27]). Both ${ }^{\rho} \mathbb{C}$ and ${ }^{\rho} \mathcal{E}(\Omega)$ are defined in the framework 
of non-standard analysis (see Definition 7.1 and Definition 7.7 below). As far as we treat $\widehat{\mathbb{C}^{\mathcal{D}_{0}}}$ and $\widehat{\mathcal{E}(\Omega)^{\mathcal{D}_{0}}}$ as modified and, we believe, improved versions of Colombeau's $\overline{\mathbb{C}}$ and $\mathcal{G}(\Omega)$, respectively, these results establish a connection between Colombeau theory and non-standard analysis.

Recall the definition of A. Robinson's field ${ }^{\rho} \mathbb{R}$ (Robinson [32]) and its complex counterpart ${ }^{\rho} \mathbb{C}$.

7.1 Definition (Robinson $\rho$-Asymptotic Numbers). Let ${ }^{*} \mathbb{R}$ and ${ }^{*} \mathbb{C}$ be the nonstandard extensions of $\mathbb{R}$ and $\mathbb{C}$, respectively in an arbitrary $\kappa$-saturated non-standard model with set of individuals $\mathbb{R}$, where $\kappa$ is an infinite cardinal. (In particular, this could be the distributional non-standard model constructed in Section 6). Let $\rho$ be a positive infinitesimal in ${ }^{*} \mathbb{R}$. Following Robinson [32], we define:

1. The sets of the $\rho$-moderate and $\rho$-negligible non-standard complex numbers are

$$
\begin{aligned}
& \mathcal{M}_{\rho}\left({ }^{*} \mathbb{C}\right)=\left\{\zeta \in{ }^{*} \mathbb{C}:|\zeta| \leq \rho^{-m} \text { for some } m \in \mathbb{N}\right\} \\
& \mathcal{N}_{\rho}\left({ }^{*} \mathbb{C}\right)=\left\{\zeta \in{ }^{*} \mathbb{C}:|\zeta|<\rho^{n} \text { for all } n \in \mathbb{N}\right\}
\end{aligned}
$$

respectively. Robinson field of complex $\rho$-asymptotic numbers is the factor ring ${ }^{\rho} \mathbb{C}=: \mathcal{M}_{\rho}\left({ }^{*} \mathbb{C}\right) / \mathcal{N}_{\rho}\left({ }^{*} \mathbb{C}\right)$. We denote by $\widehat{\zeta}$ the equivalence class of $\zeta \in \mathcal{M}_{\rho}\left({ }^{*} \mathbb{C}\right)$. For example, $\widehat{\rho}$ is the asymptotic number corresponding to $\rho$.

2. If $\mathcal{S} \subseteq{ }^{*} \mathbb{C}$, we let $\widehat{S}=\left\{\widehat{\zeta}: \zeta \in \mathcal{S} \cap \mathcal{M}_{\rho}\left({ }^{*} \mathbb{C}\right)\right\}$. If $S \subseteq \mathbb{C}$, then ${ }^{\rho} S=: \widehat{*}$ is called the $\rho$-extension of $S$. In particular, the field of Robinson real $\rho$-asymptotic numbers ${ }^{\rho} \mathbb{R}$ is the $\rho$-extension of $\mathbb{R}$, i.e. ${ }^{\rho} \mathbb{R}=\widehat{*} \mathbb{R}$. We define an order relation in ${ }^{\rho} \mathbb{R}$ as follows: Let $\widehat{\xi} \in{ }^{\rho} \mathbb{R}$ and $\widehat{\xi} \neq 0$. Then $\widehat{\xi}>0$ if $\xi>0$ in ${ }^{*} \mathbb{R}$.

3. We supply ${ }^{\rho} \mathbb{C}$ with the order topology, i.e. the product topology inherited from the order topology on ${ }^{\rho} \mathbb{R}$.

4. The valuation $v: \rho \mathbb{C} \rightarrow \mathbb{R} \cup\{\infty\}$ is defined by $v(0)=\infty$ and $v(\widehat{\zeta})=$ $\operatorname{st}(\ln |\zeta| / \ln \rho)$ if $\widehat{\zeta} \in \rho^{\rho} \mathbb{C}, \widehat{\zeta} \neq 0$. We define an ultra-norm $|\cdot|_{v}:{ }^{\rho} \mathbb{C} \rightarrow \mathbb{R}$ by the formula $|z|_{v}=e^{-v(z)}$ (under the convention that $e^{-\infty}=0$ ) and an ultra-metric by $d_{v}(a, b)=|a-b|_{v}$.

5. Let $\xi=\left(\xi_{1}, \ldots, \xi_{d}\right) \in{ }^{*} \mathbb{R}^{d}$ and $\|\xi\| \in \mathcal{M}_{\rho}\left({ }^{*} \mathbb{C}\right)$. We define $\widehat{\xi} \in{ }^{\rho} \mathbb{R}^{d}$ by $\widehat{\xi}=$ $\left(\widehat{\xi_{1}}, \ldots, \widehat{\xi_{d}}\right)$. Let $\Omega$ be an open set of $\mathbb{R}^{d}$ and $\mu(\Omega)$ be the monad of $\Omega(\# 13$ in Definition 6.1). We denote $\widehat{\mu(\Omega)}=\{\widehat{\xi}: \xi \in \mu(\Omega)\}$. 
The next result appears in (Lightstone \& Robinson ([20], p. 97).

7.2 Theorem (Principles of Permanence). Let $\mathcal{A} \subseteq{ }^{*} \mathbb{R}$ be an internal set.

(a) Overflow of $\mathcal{M}_{\rho}\left({ }^{*} \mathbb{R}\right)$ : If $\mathcal{A}$ contains arbitrarily large numbers in $\mathcal{M}_{\rho}\left({ }^{*} \mathbb{R}\right)$, then $\mathcal{A}$ contains arbitrarily small numbers in ${ }^{*} \mathbb{R} \backslash \mathcal{M}_{\rho}\left({ }^{*} \mathbb{R}\right)$.

(b) Underflow of $\mathcal{M}_{\rho}\left({ }^{*} \mathbb{R}\right) \backslash \mathcal{N}_{\rho}\left({ }^{*} \mathbb{R}\right)$ : If $\mathcal{A}$ contains arbitrarily small numbers in $\mathcal{M}_{\rho}\left({ }^{*} \mathbb{R}\right) \backslash \mathcal{N}_{\rho}\left({ }^{*} \mathbb{R}\right)$, then $\mathcal{A}$ contains arbitrarily large numbers in $\mathcal{N}_{\rho}\left({ }^{*} \mathbb{R}\right)$.

(c) Overflow of $\mathcal{N}_{\rho}\left({ }^{*} \mathbb{R}\right)$ : If $\mathcal{A}$ contains arbitrarily large numbers in $\mathcal{N}_{\rho}\left({ }^{*} \mathbb{R}\right)$, then $\mathcal{A}$ contains arbitrarily small numbers in $\mathcal{M}_{\rho}\left({ }^{*} \mathbb{R}\right) \backslash \mathcal{N}_{\rho}\left({ }^{*} \mathbb{R}\right)$.

(d) Underflow of ${ }^{*} \mathbb{R} \backslash \mathcal{M}_{\rho}\left({ }^{*} \mathbb{R}\right)$ : If $\mathcal{A}$ contains arbitrarily small numbers in $* \mathbb{R} \backslash$ $\mathcal{M}_{\rho}\left({ }^{*} \mathbb{R}\right)$, then $\mathcal{A}$ contains arbitrarily large numbers in $\mathcal{M}_{\rho}\left({ }^{*} \mathbb{R}\right)$.

7.3 Theorem (Field Properties). ${ }^{\rho} \mathbb{C}$ is an algebraically closed field, ${ }^{\rho} \mathbb{R}$ is a real closed field and we have the usual connection ${ }^{\rho} \mathbb{C}={ }^{\rho} \mathbb{R}(i)$.

Proof. The connection ${ }^{\rho} \mathbb{C}={ }^{\rho} \mathbb{R}(i)$ follows directly from the definition of ${ }^{\rho} \mathbb{C}$ and ${ }^{\rho} \mathbb{R}$. The proof that ${ }^{\rho} \mathbb{R}$ is a field can be found in (Lightstone \& Robinson [20], p.78). It follows that ${ }^{\rho} \mathbb{C}$ is also a field. Let $P(x)=x^{p}+a_{p-1} x^{p-1}+\cdots+a_{0}$ be a polynomial with coefficients in ${ }^{\rho} \mathbb{C}$ and a degree $p \geq 1$. We have $a_{n}=\widehat{\alpha_{n}}$ for some $\alpha_{n} \in \mathcal{M}_{\rho}\left({ }^{*} \mathbb{C}\right)$. We let $Q(x)=x^{p}+\alpha_{p-1} x^{p-1}+\cdots+\alpha_{0}$. Next, we observe that ${ }^{*} \mathbb{C}$ is an algebraically closed field by transfer principle (cf. Theorem 6.9 in this article or Davis [12]) since $\mathbb{C}$ is an algebraically closed field. Thus the equation $Q(\zeta)=0$ has a solution $\zeta$ in ${ }^{*} \mathbb{C}$. The estimation $|\zeta| \leq 1+\left|\alpha_{p-1}\right|+\cdots+\left|\alpha_{0}\right|$ shows that $\zeta \in \mathcal{M}_{\rho}\left({ }^{*} \mathbb{C}\right)$. Thus $P(\widehat{\zeta})=\widehat{Q(\zeta)}=\widehat{0}=0$ proving that ${ }^{\rho} \mathbb{C}$ is an algebraically closed field. It follows that $\rho \mathbb{R}$ is a real closed field as a maximal real subfield of $\rho^{\mathbb{C}}$ (Van Der Waerden [38, Chapter 11).

We turn to the connection between Robinson's theory of the field ${ }^{\rho} \mathbb{R}$ and the field of asymptotic numbers defined in Definition 4.1.

7.4 Theorem (Isomorphic Fields). Let ${ }^{*} \mathbb{R}$ and ${ }^{*} \mathbb{C}$ be the non-standard extensions of

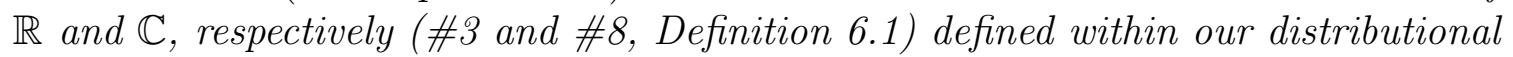
non-standard model (Section [6). Let $\rho=\left\langle R_{\varphi}\right\rangle$ be the canonical infinitesimal in ${ }^{*} \mathbb{R}$ (\#12 in Definition 6.1). Then:

(i) If $\left(A_{\varphi}\right) \in \mathbb{C}^{\mathcal{D}_{0}}$, then $\left(A_{\varphi}\right) \in \mathcal{M}\left(\mathbb{C}^{\mathcal{D}_{0}}\right)$ (Definition 4.1$)$ iff $\left\langle A_{\varphi}\right\rangle \in \mathcal{M}_{\rho}\left({ }^{*} \mathbb{C}\right)$. 
(ii) The fields $\widehat{\mathbb{C}^{\mathcal{D}_{0}}}$ and $\widehat{\mathbb{R}^{\mathcal{D}_{0}}}$ are isomorphic to ${ }^{\rho} \mathbb{C}$ and ${ }^{\rho} \mathbb{R}$, respectively, under the mapping $\widehat{A_{\varphi}} \rightarrow \widehat{\left\langle A_{\varphi}\right\rangle}$ from $\widehat{\mathbb{C}^{\mathcal{D}_{0}}}$ to ${ }^{\rho} \mathbb{C}$. This isomorphism preserves also the valuation, non-archimedean norm and ultra-metric (Definition 4.6).

(iii) The order topology and the metric topology on $\widehat{\mathbb{C}^{D_{0}}}$ are the same.

Proof. (i) $\left(A_{\varphi}\right) \in \mathcal{M}\left(\mathbb{C}^{\mathcal{D}_{0}}\right)$ iff $(\exists m \in \mathbb{N})\left\{\varphi \in \mathcal{D}_{0}:\left|A_{\varphi}\right| \leq\left(R_{\varphi}\right)^{-m}\right\} \in \mathcal{U}$ iff $(\exists m \in \mathbb{N})\left(\left|\left\langle A_{\varphi}\right\rangle\right| \leq \rho^{-m}\right)$ iff $\left\langle A_{\varphi}\right\rangle \in \mathcal{M}_{\rho}\left({ }^{*} \mathbb{C}\right)$ as required.

(ii) $\widehat{\left\langle A_{\varphi}\right\rangle}=0$ in ${ }^{\rho} \mathbb{C}$ iff $(\forall n \in \mathbb{N})\left(\left|\left\langle A_{\varphi}\right\rangle\right|<\left\langle R_{\varphi}\right\rangle^{n}\right)$ in ${ }^{*} \mathbb{C}$ iff

$$
(\forall n \in \mathbb{N})\left(\left\{\varphi \in \mathcal{D}_{0}:\left|A_{\varphi}\right|<\left(R_{\varphi}\right)^{n}\right\} \in \mathcal{U}\right),
$$

iff $\left(A_{\varphi}\right) \in \mathcal{N}\left(\mathbb{C}^{\mathcal{D}_{0}}\right)$ (Definition 4.1) iff $\widehat{A_{\varphi}}=0$ in $\widehat{\mathbb{C}^{\mathcal{D}_{0}}}$ which means that the mapping $\widehat{A_{\varphi}} \rightarrow \widehat{\left\langle A_{\varphi}\right\rangle}$ is injective. We leave to the reader to verify that this mapping preserves the ring operations.

(iii) The order topology and the metric topology on $\widehat{\mathbb{C}^{0}}$ are the same because they are the same on ${ }^{\rho} \mathbb{C}$ for any choice of ${ }^{*} \mathbb{C}$ and $\rho$ (Todorov \& Wolf [37]).

In what follows we assume a particular case of the generalized continuum hypothesis in the form $\mathfrak{c}^{+}=2^{\mathfrak{c}}$.

7.5 Corollary. Let ${ }^{*} \mathcal{R}$ be a non-standard extension of $\mathbb{R}$ in a $\mathfrak{c}^{+}$-saturated nonstandard model with set of individuals $\mathbb{R}$ such that $\operatorname{card}\left({ }^{*} \mathcal{R}\right)=\mathfrak{c}^{+}$. Let $\varepsilon$ be a positive infinitesimal in ${ }^{*} \mathcal{R}$ and let ${ }^{\varepsilon} \mathcal{C}$ and ${ }^{\varepsilon} \mathcal{R}$ be the corresponding Robinson's fields (see above). Then ${ }^{\varepsilon} \mathcal{C}$ and ${ }^{\varepsilon} \mathcal{R}$ are isomorphic to $\widehat{\mathbb{C}^{\mathcal{D}_{0}}}$ and $\widehat{\mathbb{R}^{\mathcal{D}_{0}}}$, respectively.

Proof. Let $* \mathbb{R}$ be the non-standard extension of $\mathbb{R}$ in our distributional non-standard model and let $\rho=\left\langle R_{\varphi}\right\rangle$ (\#[12 in Definition [6.1). We observe that ${ }^{*} \mathbb{R}$ is fully saturated by (Theorem $\left[6.3\right.$ ) and ${ }^{*} \mathcal{R}$ is fully saturated by assumption. Thus ${ }^{\rho} \mathbb{R}$ and ${ }^{\varepsilon} \mathcal{R}$ are isomorphic by (Todorov \& Wolf [37], p.370). It follows that ${ }^{\varepsilon} \mathcal{R}$ and $\widehat{\mathbb{R}^{\mathcal{D}_{0}}}$ are isomorphic (as required) since ${ }^{\rho} \mathbb{R}$ and $\widehat{\mathbb{R}^{\mathcal{D}_{0}}}$ are isomorphic by Theorem 7.4 .

The sets of the form $B=\left\{z \in{ }^{\rho} \mathbb{C}:|z-a|_{v} \leq b\right\}$, where $a \in{ }^{\rho} \mathbb{C}$ and $b \in \mathbb{R}_{+}$, are called closed balls in ${ }^{\rho} \mathbb{C}$. Similarly, if $a \in{ }^{\rho} \mathbb{R}$ and $b \in \mathbb{R}_{+}$, then the sets $B=\left\{z \in{ }^{\rho} \mathbb{R}:|z-a|_{v} \leq b\right\}$ are closed balls in ${ }^{\rho} \mathbb{R}$. The next result is due to W.A.J. Luxemburg ([23], p.195).

7.6 Theorem (Luxemburg). The field ${ }^{\rho} \mathbb{R}$ is spherically complete in the sense that every family of closed balls in ${ }^{\rho} \mathbb{R}$ with the finite intersection property (f.i.p.) has non-empty intersection. Consequently, the field ${ }^{\rho} \mathbb{C}$ is also spherically complete. 
We recall the definition of the algebra ${ }^{\rho} \mathcal{E}(\Omega)$ (Oberguggenberger \& Todorov[27]).

7.7 Definition ( $\rho$-Asymptotic Functions). Let $* \mathbb{R}$ and ${ }^{*} \mathcal{E}(\Omega)$ be the non-standard extensions of $\mathbb{R}$ and $\mathcal{E}(\Omega)=: \mathcal{C}^{\infty}(\Omega)$, respectively, in an arbitrary $\kappa$-saturated nonstandard model with set of individuals $\mathbb{R}$, where $\kappa$ is an infinite cardinal. (In particular, this could be the distributional non-standard model constructed in Section [6). Let $\rho$ be positive infinitesimal in ${ }^{*} \mathbb{R}$. Following (Oberguggenberger \& Todorov [27]), we define:

1. The sets of the $\rho$-moderate and $\rho$-negligible functions in ${ }^{*} \mathcal{E}(\Omega)$ are

$$
\begin{aligned}
& \mathcal{M}_{\rho}\left({ }^{*} \mathcal{E}(\Omega)\right)=\left\{f \in{ }^{*} \mathcal{E}(\Omega):\left(\forall \alpha \in \mathbb{N}_{0}^{d}\right)(\forall x \in \mu(\Omega))\left(\partial^{\alpha} f(x) \in \mathcal{M}_{\rho}\left({ }^{*} \mathbb{C}\right)\right)\right\}, \\
& \mathcal{N}_{\rho}\left({ }^{*} \mathcal{E}(\Omega)\right)=\left\{f \in{ }^{*} \mathcal{E}(\Omega):\left(\forall \alpha \in \mathbb{N}_{0}^{d}\right)(\forall x \in \mu(\Omega))\left(\partial^{\alpha} f(x) \in \mathcal{N}_{\rho}\left({ }^{*} \mathbb{C}\right)\right)\right\}
\end{aligned}
$$

respectively, where $\mu(\Omega)$ is the monad of $\Omega$ (\#13 in Definition 6.1). The differential algebra of $\rho$-asymptotic functions on $\Omega$ is the factor ring ${ }^{\rho} \mathcal{E}(\Omega)=$ : $\mathcal{M}_{\rho}\left({ }^{*} \mathcal{E}(\Omega)\right) / \mathcal{N}_{\rho}\left({ }^{*} \mathcal{E}(\Omega)\right)$. We denote by $\widehat{f}$ the equivalence class of $f \in \mathcal{M}_{\rho}\left({ }^{*} \mathcal{E}(\Omega)\right)$.

2. For any $\mathcal{S} \subseteq{ }^{*} \mathcal{E}(\Omega)$ we let $\widehat{\mathcal{S}}=\left\{\widehat{f}: f \in \mathcal{S} \cap \mathcal{M}_{\rho}\left({ }^{*} \mathcal{E}(\Omega)\right)\right\}$. If $S \subseteq \mathcal{E}(\Omega)$, the set ${ }^{\rho} S=\widehat{* S}$ is called the $\rho$-extension of $S$. The algebra ${ }^{\rho} \mathcal{E}(\Omega)$ consists of particular pointwise functions from $\widehat{\mu(\Omega)}$ into ${ }^{\rho} \mathbb{C}$ (Todorov [36]).

The next result appears in (Oberguggenberger \& Todorov[27]).

7.8 Theorem (Existence of Embedding). There exists an embedding $\Sigma_{D, \Omega}: \mathcal{D}^{\prime}(\Omega) \rightarrow$ $\rho \mathcal{E}(\Omega)$ of Colombeau type, where $D \in{ }^{*} \mathcal{E}\left(\mathbb{R}^{d}\right)$ stands for a particular non-standard delta-function (non-standard mollifier). Thus ${ }^{\rho} \mathcal{E}(\Omega)$ are special algebras of generalized functions of Colombeau's type (see the Introduction).

7.9 Remark (Non-Canonical Embedding). We should notice that the embedding $\Sigma_{D, \Omega}$ is non-canonical because the existence of $D$ is proved in [27] by saturation principle and thus $D$ cannot be defined uniquely in the terms already used in the definition of $\rho \mathcal{E}(\Omega)$. Actually, $D$ cannot be determined uniquely by any properties expressed in the language of standard or non-standard analysis; $D$ is chosen and fixed in [27] "by hand".

The next two simple lemmas provide examples of the ability of non-standard analysis to reduce the number of quantifiers.

7.10 Lemma. Let $f \in{ }^{*} \mathcal{E}(\Omega)$. Then the following are equivalent: 
(a) $(\forall K \Subset \Omega)(\exists m \in \mathbb{N})\left(\sup _{\xi \in \in^{*} K}|f(\xi)| \leq \rho^{-m}\right)$.

(b) $(\forall \xi \in \mu(\Omega))\left(f(\xi) \in \mathcal{M}_{\rho}\left({ }^{*} \mathbb{C}\right)\right)$.

Proof. (a) $\Rightarrow$ (b): Suppose that $\xi \in \mu(\Omega)$ and let $\operatorname{st}(\xi)=s$. Since $s \in \Omega$ and $\Omega$ is open, there exists an open relatively compact subset $\mathcal{O}$ of $\Omega$ which contains $s$ and such that $\overline{\mathcal{O}} \subset \Omega$. So, we have $\xi \in{ }^{*} K$, where $K=\overline{\mathcal{O}}$. Thus $\sup _{\eta \in *^{*} K}|f(\eta)| \leq \rho^{-m}$ for some $m \in \mathbb{N}$ (by assumption) implying $f(\xi) \in \mathcal{M}_{\rho}\left({ }^{*} \mathbb{C}\right)$ as required.

(a) $\Leftarrow($ b): Let $K$ be a compact subset of $\Omega$ and suppose (on the contrary) that $(\forall m \in \mathbb{N})\left(\sup _{\xi \in \in^{*} K}|f(\xi)|>\rho^{-m}\right)$. Next, we observe that the set $\mathcal{A}=:\{m \in$ $\left.{ }^{*} \mathbb{N}: \sup _{\xi \in{ }^{*} K}|f(\xi)|>\rho^{-m}\right\}$ is internal by internal definition principle (Theorem 6.12). Also, $\mathcal{A}$ contains $\mathbb{N}$ by assumption and thus $\mathcal{A}$ contains an infinitely large number $\nu \in$ ${ }^{*} \mathbb{N}$ by overflow of $\mathcal{F}\left({ }^{*} \mathbb{R}\right.$ ) (cf. Theorem 6.5) in this article or Capiński \& Cutland [4, p.24). Thus we have $\sup _{\xi \in \in^{*} K}|f(\xi)|>\rho^{-\nu}$. On the other hand, we have $\left|f\left(\xi_{0}\right)\right|>\rho^{-\nu}$ for some $\xi_{0} \in{ }^{*} K$ by transfer principle (cf. Theorem 6.9 in this article or Davis [12]), contradicting (a), since ${ }^{*} K \subset \mu(\Omega)$ by Theorem 6.6 .

7.11 Lemma. Let $f \in{ }^{*} \mathcal{E}(\Omega)$. Then the following are equivalent:

(a) $(\forall K \Subset \Omega)(\forall n \in \mathbb{N})\left(\sup _{\xi \in \in^{*} K}|f(\xi)|<\rho^{n}\right)$.

(b) $(\forall \xi \in \mu(\Omega))\left(f(\xi) \in \mathcal{N}_{\rho}\left({ }^{*} \mathbb{C}\right)\right)$.

Proof. The proof is very similar to the proof of the above lemma and we leave it to the reader.

7.12 Theorem (Isomorphic Algebras). Let ${ }^{*} \mathcal{E}(\Omega)$ be the non-standard extension of $\mathcal{E}(\Omega)$ (\#胥, Definition 6.1) in the distributional ultrapower non-standard model constructed in Section 6. Let $\rho=\left\langle R_{\varphi}\right\rangle$ be the canonical infinitesimal in * ${ }^{*}$ (\#10 in Definition 6.1). Then:

(i) If $\left(f_{\varphi}\right) \in \mathcal{E}(\Omega)^{\mathcal{D}_{0}}$, then $\left(f_{\varphi}\right) \in \mathcal{M}\left(\mathcal{E}(\Omega)^{\mathcal{D}_{0}}\right)$ (Definition 4.9) iff $\left\langle f_{\varphi}\right\rangle \in \mathcal{M}_{\rho}\left({ }^{*} \mathcal{E}(\Omega)\right.$ ).

(ii) The differential algebras $\widehat{\mathcal{E}(\Omega)^{\mathcal{D}_{0}}}$ and ${ }^{\rho} \mathcal{E}(\Omega)$ are isomorphic under the mapping $\widehat{f_{\varphi}} \rightarrow \widehat{\left\langle f_{\varphi}\right\rangle}$ from $\widehat{\mathcal{E}(\Omega)^{\mathcal{D}_{0}}}$ to ${ }^{\rho} \mathcal{E}(\Omega)$.

Proof. In view of the previous two lemmas, the proof of this theorem is almost identical to the proof of Theorem 7.4 and we leave it to the reader. 


\section{The Hahn-Banach Extension Principle for Asymp- totic Functionals}

In this section we show that a Hahn-Banach extension principle holds for continuous asymptotic functionals, i.e. linear continuous functionals defined on vector spaces over the field ${ }^{\rho} \mathbb{C}$ taking values also in ${ }^{\rho} \mathbb{C}$ (Corollary 8.3). This result is based on the spherical completeness of ${ }^{\rho} \mathbb{C}$ (Luxemburg [23], p.195 or Theorem 7.6] in this article) and a result due to (Ingleton [15]). Here ${ }^{\rho} \mathbb{C}$ is Robinson's field (Definition 7.1) within an arbitrary non-standard model with individuals $\mathbb{R}$ and $\rho$ is an arbitrary positive infinitesimal in ${ }^{*} \mathbb{R}$. Consequently, the results in this section hold as well for linear continuos functionals with values in $\widehat{\mathbb{C}^{\mathcal{D}_{0}}}$ (Definition 4.1) since $\widehat{\mathbb{C}^{\mathcal{D}_{0}}}$ is isomorphic to a field of the form ${ }^{\rho} \mathbb{C}$ (Theorem 7.4).

The rings of Colombeau generalized numbers $\overline{\mathbb{R}}$ and $\overline{\mathbb{C}}$ (Colombeau [6], pp.136) are also spherically complete, and a result similar to Theorem 8.1 appears in E. Mayerhofer's thesis [24], where $\mathbb{K}$ (see below) is a field which is a (proper) subring of $\overline{\mathbb{C}}$. Also E. Mayerhofer raised the question whether or not it is possible to generalize his result to the whole rings $\overline{\mathbb{R}}$ and $\overline{\mathbb{C}}$ (cf. Conjecture 3.11 in Mayerhofer 24]). Later H. Vernaeve [39] proved that a such generalization is impossible. Thus Corollary 8.3 at the end of this section does not have a counterpart in Colombeau theory. We look upon this fact as one more piece of evidence supporting the point (advocated for a long time by the first author of this article) that Robinson's field ${ }^{\rho} \mathbb{C}$ along with the algebra of asymptotic functions ${ }^{\rho} \mathcal{E}(\Omega)$ are better alternatives to the ring of Colombeau's generalized scalars $\overline{\mathbb{C}}$ and Colombeau's algebra of generalized functions $\mathcal{G}(\Omega)$ for the purpose of non-linear theory of generalized functions and functional analysis in general.

The reader might observe some similarity between the field ${ }^{\rho} \mathbb{R}$ (and ${ }^{\rho} \mathbb{C}$ as well) and the fields of the $p$-adic numbers $\mathbb{Q}_{p}$ (Ingleton [15]). This similarity is due to the fact that ${ }^{\rho} \mathbb{R},{ }^{\rho} \mathbb{C}$ and $\mathbb{Q}_{p}$ are all ultra-metric spaces. For a discussion on this topic we refer to (Luxemburg [23]). We should mention, however, that the fields ${ }^{\rho} \mathbb{R},{ }^{\rho} \mathbb{C}$ and $\mathbb{Q}_{p}$ are quite different from each other. For example, each ${ }^{\rho} \mathbb{R}\left(\right.$ just like ${ }^{*} \mathbb{R}$ ) is a real closed, and thus, a totally ordered field. Also each ${ }^{\rho} \mathbb{C}$ (just like ${ }^{*} \mathbb{C}$ ) is a algebraically closed field. In contrast, the fields $\mathbb{Q}_{p}$ are neither algebraically closed, nor real closed fields. In fact $\mathbb{Q}_{p}$ are not even real fields, that is to say that $\mathbb{Q}_{p}$ are non-orderable. Recall that a field $\mathbb{K}$ is orderable iff $\mathbb{K}$ is real in the sense that equations of the form $x_{1}^{2}+x_{2}^{2}+\cdots+x_{n}^{2}=0$ admit only trivial solutions $x_{1}=x_{2}=\cdots=x_{n}=0$ in $\mathbb{K}$ (Van Der Waerden [38], Chapter 11). Neither of the fields $\mathbb{Q}_{p}$ has this property (Ribenboim [30], pp.144-145).

We start with some preliminaries: 
1. Let $\mathbb{K}$ be a subfield of ${ }^{\rho} \mathbb{C}$. Let $V$ be a vector space over $\mathbb{K}$ and let $\|\cdot\|_{v}: V \rightarrow \mathbb{R}$ be a ultra-norm on $V$. The latter means that for every $x, y \in V$ and $c \in \mathbb{K}$, we have: (a) $\|x\|_{v} \geq 0$ and $\|x\|_{v}=0$ occurs only if $x=0$; (b) $\|c x\|_{v}=|c|_{v}\|x\|_{v}$, where $|c|_{v}$ is defined in \#4 in Definition [7.1, (c) $\|x+y\|_{v} \leq \max \left\{\|x\|\left\|_{v},\right\| y \|_{v}\right\}$ (ultra-norm inequality). We denote by $\left(V, \mathbb{K},\|\cdot\|_{v}\right)$ the corresponding ultranormed vector space over $\mathbb{K}$. Notice, in particular, that if $V$ is an inner vector space over $\mathbb{K}$, then the formula $\|x\|_{v}=\sqrt{|(x, x)|_{v}}$ defines a ultra-norm on $V$. Also, if $\mathbb{K}$ is an algebraically closed (or real closed) field, then the formula $\|x\|_{v}=|\sqrt{(x, x)}|_{v}$ also produces a ultra-norm on $V$.

2. Let $V^{*}$ be the algebraic dual of $V$, i.e. the vector space over $\mathbb{K}$ of all linear functionals $T: V \rightarrow \mathbb{K}$. We shall use the same notation, $\|\cdot\|_{v}$, for the nonarchimedean norm $\|\cdot\|_{v}: V^{*} \rightarrow \mathbb{R} \cup\{\infty\}$ inherited from $V$ by duality, i.e. $\|F\|_{v}=\sup _{\substack{x \in V \\\|x\|_{v}=1}}|T(x)|_{v}$.

3. $T \in V^{*}$ is called continuous if $\|T\|_{v} \in \mathbb{R}$ (i.e. if $\|T\|_{v} \neq \infty$ ). We denote by $V^{\prime}$ the vector space over $\mathbb{K}$ of all continuous functionals in $V^{*}$. Thus $|T(x)|_{v} \leq$ $\|T\|_{v}\|x\|_{v} \in \mathbb{R}$ holds for all $T \in V^{\prime}$ and all $x \in V$.

Here is our Hahn-Banach extension principle.

8.1 Theorem (Hahn-Banach). Let $\mathbb{K}$ be a subfield of ${ }^{\rho} \mathbb{C}$ which is spherically complete under the ultrametric on ${ }^{\rho} \mathbb{C}$. Let $\left(V, \mathbb{K},\|\cdot\|_{v}\right)$ be an ultra-normed vector space over $\mathbb{K}$. Let $U$ be a $\mathbb{K}$-linear subspace of $V$. Then every functional $T \in U^{\prime}$ can be extended (non-uniquely) to a functional $M \in V^{\prime}$ such that $\|T\|_{v}=\|M\|_{v}$.

Proof. The above theorem is a particular case of A. W. Ingleton's result in [15]. A similar independent proof follows: If $U=V$ there is nothing to prove. Suppose $x_{0} \in V \backslash U$ and let $U+\mathbb{K} x_{0}=\left\{x+c x_{0}: x \in U, c \in \mathbb{K}\right\}$. Our first goal is to extend $T$ to a functional $S$ on $U+\mathbb{K} x_{0}$. We let $S\left(x+c x_{0}\right)=T(x)+c S\left(x_{0}\right)$. To complete this definition we need to prescribe a value $S\left(x_{0}\right)=y_{0}$ which preserves the $v$-norm of $T$. For any $x \in U$ we define $R(x)=:\|T\|_{v}\left\|x-x_{0}\right\|_{v}$ and the closed ball $\mathcal{B}_{x}=\left\{y \in \mathbb{K}:|y-T(x)|_{v} \leq R(x)\right\}$. The family $\left\{\mathcal{B}_{x}\right\}_{x \in U}$ has the finite intersection property. Indeed, suppose that $x_{1}, x_{2} \in U$ and $x_{1} \neq x_{2}$. By involving the ultra-norm inequality, we obtain: $\left|T\left(x_{1}\right)-T\left(x_{2}\right)\right|_{v} \leq\|T\|_{v}\left\|x_{1}-x_{2}\right\|_{v} \leq$ $\|T\|_{v} \max \left\{\left\|x_{1}-x_{0}\right\|_{v},\left\|x_{0}-x_{2}\right\|_{v}\right\}=\max \left\{R\left(x_{1}\right), R\left(x_{2}\right)\right\}$. If $R\left(x_{1}\right) \leq R\left(x_{2}\right)$, then $T\left(x_{1}\right) \in \mathcal{B}_{x_{2}}$. If $R\left(x_{1}\right) \geq R\left(x_{2}\right)$, then $T\left(x_{2}\right) \in \mathcal{B}_{x_{1}}$. Since $T\left(x_{1}\right)$ and $T\left(x_{2}\right)$ are the centers of the balls $\mathcal{B}_{x_{1}}$ and $\mathcal{B}_{x_{2}}$, respectively, it follows that either $T\left(x_{1}\right) \in \mathcal{B}_{x_{1}} \cap \mathcal{B}_{x_{2}}$, or $T(x) \in \mathcal{B}_{x_{1}} \cap \mathcal{B}_{x_{2}}$. In either case we have $\mathcal{B}_{x_{1}} \cap \mathcal{B}_{x_{2}} \neq \varnothing$, as required. Notice that 
the latter implies either $\mathcal{B}_{x_{1}} \subset \mathcal{B}_{x_{2}}$, or $\mathcal{B}_{x_{1}} \supset \mathcal{B}_{x_{2}}$ due to the ultra-norm inequality, hence the argument can be repeated for any finite number of elements in $U$. Thus there exists $y_{0} \in \bigcap_{x \in U} \mathcal{B}_{x}$ since $\mathbb{K}$ is spherically complete by assumption. We let $S\left(x_{0}\right)=y_{0}$ and the definition of $S$ is complete. Clearly $S$ is an extension of $T$. To show the preservation of the norm of $T$, observe that $\|T\|_{v} \leq\|S\|_{v}$ trivially. To show $\|T\|_{v} \geq\|S\|_{v}$, suppose that $x-c x_{0} \in U+\mathbb{K} x_{0}$. Notice that $\left|y_{0}-T(x)\right|_{v} \leq R_{x}$ for all $x \in U$, by the choice of $y_{0}$. With this in mind, we estimate $\left|S\left(x-c x_{0}\right)\right|_{v}=$ $|c|_{v}\left|S\left(x / c-x_{0}\right)\right|_{v}=|c|_{v}\left|S(x / c)-S\left(x_{0}\right)\right|_{v}=|c|_{v}\left|T(x / c)-y_{0}\right|_{v} \leq|c|_{v} R(x / c)=$ $|c|_{v}\|T\|_{v}\left\|x / c-x_{0}\right\|_{v}=\|T\|_{v}\left\|x-c x_{0}\right\|_{v}$. Thus $\|T\|_{v}=\|S\|_{v}$, as required. The rest of the proof is a typical application of Zorn's lemma. Let $\mathcal{L}_{T}$ denote the set of all extensions of $T$ which preserves the $v$-norm of $T$. Notice that $\mathcal{L}_{T} \neq \varnothing$ since $S \in \mathcal{L}_{T}$. If $T_{1}, T_{2} \in \mathcal{L}_{T}$ and if $T_{2}$ is an extension of $T_{1}$, we shall write $T_{1} \prec T_{2}$ and also $T_{1} \vee T_{2}=T_{2}$. Let $\mathcal{L}$ be a subset of $\mathcal{L}_{T}$ which is totally ordered under $\prec$. We observe that $\bigvee_{L \in \mathcal{L}} L \in \mathcal{L}_{T}$. Thus, by Zorn's lemma, $\mathcal{L}_{T}$ has maximal elements; let $M$ be a such maximal element of $\mathcal{L}_{T}$. The functional $M$ is an extension of $T$ and $\|T\|_{v}=\|M\|_{v}$, by the definition of $\mathcal{L}_{T}$. The domain of $M$ should be $V$. Suppose (on the contrary) that the domain $\operatorname{dom}(M)$ of $M$ is a proper subset of $V$. Then we can, as before, choose $z_{0} \in V \backslash \operatorname{dom}(M)$ and extend $M$ to $\operatorname{dom}(M)+\mathbb{K} z_{0}$, contradicting the maximality of $M$.

8.2 Example (Power Series). Let $\mathbb{C}\langle x\rangle$ be the Levi-Civita field consisting of all formal series of the form $\sum_{n=0}^{\infty} a_{n} x^{r_{n}}$, where $a_{n} \in \mathbb{C}$ and $\left(r_{n}\right)$ is a strictly increasing unbounded sequence in $\mathbb{R}$ (Levi-Civita [18]). The field $\mathbb{C}\langle x\rangle$ is isomorphic to the field of algebraic functions in one variable in the sense that $\mathbb{C}\langle x\rangle$ is an algebraic closure of the field of rational functions $\mathbb{C}(x)$. The field $\mathbb{C}\langle x\rangle$ is spherically complete (Luxemburg [23]) and it can be embedded in ${ }^{\rho} \mathbb{C}$ by the mapping $\sum_{n=0}^{\infty} a_{n} x^{r_{n}} \rightarrow$ $\sum_{n=0}^{\infty} a_{n} \rho^{r_{n}}$ (cf. Robinson [32] or Lightstone \& Robinson [20]). The above HahnBanach extension principle holds for its image $\mathbb{K}=\mathbb{C}\langle\rho\rangle$. For more examples of spherically complete algebraically closed and real closed subfields $\mathbb{K}$ of ${ }^{\rho} \mathbb{C}$, we refer to (Todorov \& Wolf [37]).

The next result does not have a counterpart in Colombeau theory (Vernaeve [39]) since $\overline{\mathbb{R}}$ and $\overline{\mathbb{C}}$ are rings with zero divisors.

8.3 Corollary (The Case $\left.\mathbb{K}={ }^{\rho} \mathbb{C}\right)$. Let $\left(V,{ }^{\rho} \mathbb{C},\|\cdot\|_{v}\right)$ be a ultra-normed vector space over the field ${ }^{\rho} \mathbb{C}$. Let $U$ be a ${ }^{\rho} \mathbb{C}$-linear subspace of $V$. Then every functional $T \in U^{\prime}$ can be extended (non-uniquely) to a functional $M \in V^{\prime}$ such that $\|T\|_{v}=\|M\|_{v}$. A similar result holds about any ultra-normed vector space $\left(V,{ }^{\rho} \mathbb{R},\|\cdot\|_{v}\right)$ over the field $\rho \mathbb{R}$. 
Proof. Since both ${ }^{\rho} \mathbb{C}$ and ${ }^{\rho} \mathbb{R}$ are spherically complete fields (cf. Luxemburg [22], p. 195 or Theorem 7.6 in this article), we can apply the above theorem for $\mathbb{K}={ }^{\rho} \mathbb{C}$ and $\mathbb{K}=\rho \mathbb{R}$, respectively.

Acknowledgment: The authors thank Michael Oberguggenberger for the useful discussion on preliminary versions of this article. The first author is grateful to the colleagues at the University of Vienna and especially to Michael Kunzinger for the hospitality and financial support in the Spring of 2006, where the work on this article began.

\section{References}

[1] Guy Berger, Non-Standard Analysis, multiplication of Schwartz distributions, and delta-like solution of Hopf's Equation, Master Thesis \# LD729. 6. S52 M3 B47 (2005) in R.E. Kennedy Library of the California Polytechnic State University, San Luis Obispo, CA-93407.

[2] H. A. Biagioni, A Nonlinear Theory of Generalized Functions, Lecture Notes in Mathematics, Springer Verlag, Vol. 1421, XII, 1990.

[3] H. Bremermann, Distributions, Complex Variables, and Fourier Transforms, Addison-Wesley Publ. Co., Inc., Palo Alto, 1965.

[4] M. Capiński and N. J. Cutland, Nonstandard Methods for Stochastic Fluid Mechanics, World Scientific, Singapore-New Jersey-London-Hong Kong, 1995.

[5] C. C. Chang and H. J. Keisler, Model Theory, Studies in Logic and the Foundations of Mathematics, Vol. 73, Elsevier, Amsterdam, 1998.

[6] J. F. Colombeau, New Generalized Functions and Multiplication of Distributions, North-Holland Math. Studies 84, 1984.

[7] J. F. Colombeau, Elementary Introduction to New Generalized Functions, North Holland, Amsterdam 1985.

[8] J.F. Colombeau, A.Y. Le Roux, Multiplication of distributions in elasticity and hydrodynamics, J. Math. Phys. 29 (1988), 315 - 319.

[9] J. F. Colombeau, Multiplication of Distributions, Bull.A.M.S. 23, 2, 1990, pp. 251-268. 
[10] J. F. Colombeau, Multiplication of Distributions; A Tool in Mathematics, Numerical Engineering and Theoretical Physics, Lecture Notes in Mathematics, 1532, Springer- Verlag, Berlin, 1992.

[11] J. F. Colombeau, A. Gsponer and B. Perrot, Nonlinear generalized functions and Heisenberg-Pauli foundations of quantum field theory, a preprint, arXiv: 0705.2396v1 [math-ph], 16 May, 2007.

[12] M. Davis, Applied Nonstandard Analysis, Dover Publications, Inc., Mineola, New York, 2005.

[13] M. Grosser, Eva Farkas, M. Kunzinger and R. Steinbauer, On the Foundations of Nonlinear Generalized Functions I and II, Memoirs of the AMS, Vol. 153, Number 729, American Mathematical Society, 2001.

[14] M. Grosser, M. Kunzinger, M. Oberguggenberger and R. Steinbauer, Geometric Theory of Generalized Functions withy Applications to General Relativity, Vol. 537 of Mathematics and Its Applications, Kluwer Academic Publishers, Dordrecht, 2001.

[15] A. W. Ingleton, The Hahn-Banach theorem for non-archimedean valued fields, Proc. Cambridge Phil. Soc. 48 (1952), pp. 41-45.

[16] A. Kaneko, Introduction to Hyperfunctions, Kluwer Acad. Publ., Dordrecht 1988.

[17] J. L. Kelley, General Topology, Prindle, Springer-Verlag, New York, Berlin, Heidelberg, Tokyo, 1975.

[18] T. Levi-Civita, Sugli Infiniti ed Infinitesimi Attuali Quali Elementi Analitici (1892-1893), Opere Mathematiche, vol. 1, Bologna (1954), p. 1-39.

[19] Li Bang-He, Non-Standard Analysis and multiplication of disributions, Sci. Sinica, 21 (1978), pp. 561-585.

[20] A. H. Lightstone and A. Robinson, Nonarchimedean Fields and Asymptotic Expansions, North-Holland, Amsterdam, 1975.

[21] T. Lindstrøm, An invitation to nonstandard analysis, in: Nonstandard Analysis and its Applications, N. Cutland (Ed), Cambridge U. Press, 1988, pp. 1-105. 
[22] W. A. J. Luxemburg, Non-Standard Analysis: Lectures on A. Robinson's Theory of Infinitesimals and Infinitely Large Numbers, California Institute of Technology, Pasadena, California, 1962 (Second Edition, 1973).

[23] W. A. J. Luxemburg, On a class of valuation fields introduced by Robinson, Israel J. Math. 25 (1976), pp. 189-201.

[24] E. Mayerhofer, The wave equation on singular space-times, A Ph.D. Thesis, Faculty of Mathematics, University of Vienna, 1090 Vienna, Austria.

[25] M. Oberguggenberger, Multiplication of Distributions and Applications to Partial Differential Equations, Pitman Research Notes Math., 259, Longman, Harlow, 1992.

[26] M. Oberguggenberger, Contributions of nonstandard analysis to partial differential equations, in: Developments in Nonstandard Mathematics (Eds. N.J. Cutland, V. Neves, F. Oliveira and J. Sousa-Pinto), Longman Press, Harlow, 1995, pp. 130-150.

[27] M. Oberguggenberger and T. Todorov, An embedding of Schwartz distributions in the algebra of asymptotic functions, Int'l. J. Math. and Math. Sci. 21 (1998), pp. 417-428.

[28] M. Oberguggenberger and H. Vernaeve, Internal sets and internal functions in Colombeau theory, to appear in Journal of Mathematical Analysis and Applications.

[29] V. Pestov, On a valuation field invented by A. Robinson and certain structures connected with it, Israel J. Math. 74 (1991), pp. 65-79.

[30] P. Ribenboim, The Theory of Classical Valuations, Springer Monographs in Mathematics, Springer-Verlag, 1999.

[31] A. Robinson, Nonstandard Analysis, North Holland, Amsterdam, 1966.

[32] A. Robinson, Function theory on some nonarchimedean fields, Amer. Math. Monthly 80 (6), Part II: Papers in the Foundations of Mathematics (1973), pp. 87-109.

[33] S. Salbany and T. Todorov, Nonstandard Analysis in Point-Set Topology, Lecture Notes No. 666, 1998 (52 pages) of Erwing Schrödinger Institute for Mathematical Physics, Vienna (ftp at ftp.esi.ac.at, URL: http://www.esi.ac.at). 
[34] L. Schwartz, Sur l'impossibilité de la multiplication des distributions, C.R.Acad.Sci., Paris 239 (1954), pp. 847-848.

[35] K. D. Stroyan and W. A. J. Luxemburg, Introduction to the Theory of Infinitesimals, Academic Press, New York, 1976.

[36] T. Todorov, Pointwise Values and Fundamental Theorem in the Algebra of Asymptotic Functions, in Non-Linear Theory of Generalized Functions (Eds: M. Grosser, Günther Hörmann, M. Kunzinger and M. Oberguggenberger), Chapman \& Hall/CRC Research Notes in Mathematics, 401, 1999, pp. 369-383.

[37] T. D. Todorov and R. S. Wolf, Hahn Field Representation of A. Robinson's Asymptotic Numbers, in Nonlinear Algebraic Analysis and Applications, Proceedings of the ICGF 2000 (Edited by A. Delcroix, M. Hasler, J.A. Marti, V. Valmorin), 2004 Cambridge Scientific Publishers, p. 357-374, arXiv:math.AC/0601722.

[38] B. L. Van Der Waerden, Modern Algebra, Ungar Publishing, New York, third printing, 1964.

[39] H. Vernaeve, Ideals in the ring of Colombeau generalized numbers, preprint, arXiv:0707.0698,

[40] V. Vladimirov, Generalized Functions in Mathematical Physics, Mir-Publisher, Moscow, 1979. 